\begin{tabular}{|c|l|}
\hline Title & Langevin equations and causal analysis \\
\hline Author(s) & Okabe, Y asunori \\
\hline Citation & Hokkaido University Preprint Series in Mathematics, 148, 2-42 \\
\hline Issue Date & 1992-05 \\
\hline DOI & 10.14943/83292 \\
\hline Doc URL & http://hdl.handle.net/2115/68894 \\
\hline Type & bulletin (article) \\
\hline File Information & pre148.pdf \\
\hline
\end{tabular}

Instructions for use 
Langevin equations and Causal analysis

\author{
Yasunori Okabe
}

Series $\sharp 148$. May 1992 


\section{HOKKAIDO UNIVERSITY PREPRINT SERIES IN MATHEMATICS}

$\sharp 122$ : Y.-G. Chen, Blow-up solutions to a finite difference analogue of $u_{t}=\Delta u+u^{1+\alpha}$ in $N$-dimensional balls, 31 pages. 1991.

$\sharp 123$ : A. Arai, Fock-space representations of the relativistic supersymmetry algebra in the two-dimensional spacetime, 13 pages. 1991.

\#124: S. Izumiya, The theory of Legendrian unfoldings and first order differential equations, 16 pages. 1991.

$\sharp 125$ : T. Hibi, Face number inequalities for matroid complexes and Cohen-Macaulay types of Stanley-Reisner rings of distributive lattices, 17 pages. 1991.

$\sharp 126$ : S. Izumiya, Completely integrable holonomic systems of first order differential equations, 35 pages. 1991.

$\sharp 127$ : $\quad$ G. Ishikawa, S. Izumiya and K. Watanabe, Vector fields near a generic submanifold, 9 pages. 1991.

$\sharp 128$ : A. Arai, I. Mitoma, Comparison and nuclearity of spaces of differential forms on topological vector spaces, 27 pages. 1991.

$\sharp 129$ : K. Kubota, Existence of a global solution to a semi-linear wave equation with initial data of non-compact support in low space dimensions, 53 pages. 1991.

$\sharp 130$ : S. Altschuler, S. Angenent and Y. Giga, Mean curvature flow through singularities for surfaces of rotation, 62 pages. 1991.

$\sharp 131$ : M. Giga, Y. Giga and H. Sohr, $L^{p}$ estimates for the Stokes system, 13 pages. 1991.

$\#$ 132: Y. Okabe, T. Ootsuka, Applications of the theory of $\mathrm{KM}_{2} \mathrm{O}$-Langevin equations to the non-linear prediction problem for the one-dimensional strictly stationary time series, 27 pages. 1992.

$\sharp 133$ : Y. Okabe, Applications of the theory of $\mathrm{KM}_{2} \mathrm{O}$-Langevin equations to the linear prediction problem for the multi-dimensional weakly stationary time series, 22 pages. 1992.

$\sharp 134: \quad$ P. Aviles, Y. Giga and N. Komuro, Duality formulas and variational integrals, 22 pages. 1992.

$\sharp 135$ : S. Izumiya, The Clairaut type equation, 6 pages. 1992.

\# 136: S. Izumiya, Singular solutions of first order differential equations, 6 pages. 1992.

\#137: S. Izumiya, W.L. Marar, The Euler characteristic of a generic wave front in a 3-manifold, 6 pages. 1992.

$\sharp 138$ : S. Izumiya, W.L. Marar, The Euler characteristic of the image of a stable mapping from a closed $n$-manifold to a $(2 n-1)$-manifold, 5 pages. 1992.

$\sharp 139$ : Y. Giga, Z. Yoshida, A bound for the pressure integral in a plasma equilibrium, 20 pages. 1992.

$\sharp 140$ : S. Izumiya, What is the Clairaut equation ?, 13 pages. 1992.

$\sharp 141$ : H. Takamura, Weighted deformation theorem for normal currents, 27 pages. 1992.

$\sharp 142$ : T. Morimoto, Geometric structures on filtered manifolds, 104 pages. 1992.

$\sharp$ 143: G. Ishikawa, T. Ohmoto, Local invariants of singular surfaces in an almost complex four-manifold, 9 pages. 1992 .

$\sharp 144: \quad$ K. Kubota, K. Mochizuki, On small data scattering for 2-dimensional semilinear wave equations, 22 pages. 1992.

$\sharp 145: \quad$ T. Nakazi, K. Takahashi, Hyponormal Toeplitz operators and extremal problems of Hardy spaces, 30 pages. 1992.

\# 146: N. Hayashi, T. Ozawa, Remarks on nonlinear Schrödinger equations in one space dimension, 10 pages. 1992.

$\sharp 147$ : $\quad$ M. Sato, Interface evolution with Neumann boundary condition, 16 pages. 1992. 


\title{
Langevin equations and Causal analysis
}

\author{
Yasunori Okabe \\ Department of Mathematics \\ Faculty of Science \\ Hokkaido University \\ Sapporo 060, JAPAN
}

\section{$\oint 1$. Introduction}

With Einstein's theory of Brownian motion ([6]) and Langevin's theory of stochastic differential equations ([16]) in statistical physics as its origin, the present theory of diffusion processes in probability theory teaches us the following: the infinitesimal generators of semigroups associated with the diffusion processes and the time evolution of themselves can be characterized from the qualitative nature of diffusion property as Kolmogorov's second order differential equations of elliptic type and Ito's stochastic differential equations of Markovian type, respectively.

In the essay [On a Langevin equation] which appeared ten years ago ([24]), we have introduced the qualitative property of $T$-positivity, which is broader than the one of diffusion property, from the requirement in quantum field theory and discussed that this qualitative property determines the $[\alpha, \beta, \gamma]$-Langevin equation ( $\equiv$ model) in the latter sense stated above. This study was started with an aim to clarify a mathematical structure of the fluctuation-dissipation theorem which is one of the fundamental principles in non-equilibrium statistical physics.

The investigation that cannot but throw doubt about Einstein's theory, however, has been already presented in the 1960's. In the study in statistical physics by Alder and Wainwright ([2]), a computer simulation of a hydrodynamic model compounded of a hard sphere and a hard disk, it has been reported that the motion of the hard sphere has the so-called Alder-Wainwright effect, that is, that the velocity correlation function decreases not exponentially, but polynomially.

The study to clarify such an Alder-Wainwright effect from the viewpoint of statistical 
physics, was proceeded with an application of Kubo's linear response theory ([13]) to the study by Stokes and Boussinesq $([41],[3])$ made in the late nineteen century before the one by Einstein and Langevin. The Alder-Wainwright effect is now verified in both fields of the theory and the experiment $([43],[15],[40])$. In the course of these researches, it has been recognized that the model for the velocity of Brownian motion with Alder-Wainwright effect can be described as a stationary solution of Stokes-Boussinesq-Langevin equation whose approximate accuracy is thermodynamically better than the classical Langevin equation, that is, Ornstein-Uhlenbeck's Langevin equation.

We have perceived that this stationary solution has $T$-positivity from the point of view of the theory of stochastic processes and felt keenly that it is necessary to release the conditions posed in [20]-[24] as a requirement from statistical physics. This gave us a generative power which developed our previous studies. In both the continuous case and the discrete case, we have obtained KMO-Langevin equations describing the time evolution of weakly stationary processes with $T$-positivity as a general form of Stokes-Boussinesq-Langevin equation and clarified a mathematical structure of Alder-Wainwright effect ([27]-[34]). Thus $[\alpha, \beta, \gamma]$-Langevin equations in [22] changed their form into KMO-Langevin equations which have sufficient significance from the viewpoint of both probability theory and statistical physics. Furthermore in the discrete case, with an aim to obtain certain algorithm available to computer science in technology, social science and so on, KMO-Langevin equations with infinite delay drift terms offered their field of researches to $\mathrm{KM}_{2} \mathrm{O}$-Langevin equations with finite delay drift terms which characterize the local and weak stationarity of stochastic processes with discrete time $([35],[39],[38])$. The guidance principle therein is the following philosophy:

FDP(Fluctuation-Dissipation Principle): In order to clarify natural and social phenomena with random time evolution, we should not do a priori argument in model building. At first we take notice of certain qualitative nature (e.g., local and weak stationarity) to characterize stochastic models to which we want to apply the theory of stochastic processes and then we check its property from a given data. It is important for us to take out a random force giving rise to its complexity from the data, according to the fluctuationdissipation theorem. 
This FDP becomes really effective in causal analysis. If certain causal relation between two kinds of data which vary randomly according to the change of time were asserted on the basis of certain models constructed deductively, we would not be able to have any objective persuasive power. Thus we propose a stationary test-Test(S)-checking the local and weak stationarity of time series formed by a given data and a causal test-Test(CS)judging the existence and direction of causal relation between two kinds of given data: At first we check the local and weak stationarity for stochastic process with a given data as its realization on the bassis of the theory of $\mathrm{KM}_{2} \mathrm{O}$-Langevin equation (Test(S)). If the given data does not pass Test(S), we apply appropriate injective transformations to the original data and then examine Test(S) for the new data. We can proceed to Test(CS) only after passing Test(S), because our Test(CS) is effective only for data passing Test(S).

However, the causal relation among things becomes a serious problem when certain abnormal affairs occur. For example, certain abnormal phenomena such as the change for the warmth, the earthquake, the eruption of volcanos and so on in the earth environment and certain finantial panics such as slump in shares in the economic world will destroy the local and weak stationarity for time series. Thus we need some effective transfomations which reproduce the local and weak stationarity. For that purpose, we consider the following three kinds of transformations:(i) differnce;(ii) logarithm (for positive data);(ii) arctangent. The important fact lies in that if these transformed data pass both Test(S) and Test(CS), then we can objectively assert the existence and direction of causal relation among given original data, taking account particularly that (ii) and (iii) have inverse transformations.

In this essay, we shall present (dissipate) our studies (fluctuation) for these ten years after the previous essay [24]. We would like to look for certain constructive criticisms from both pure mathematicians and applied mathematicians to make our guidance priciple FDP more and more powerful.

\section{$\S 2$. Stokes-Boussinesq-Langevin equation}

We shall consider a model which manifests the motion of a hard sphere of radius $r$ and mass $m$ moving with velocity $X(t)$ at time $t$ in a fluid with visicosity $\eta$ and density $\rho$, subject to a random force $W(t)$ and a drag force $F(t)$ at time $t$. Since then Newton's 
equation becomes

$$
m \dot{X}(t)=-F(t)+W(t) \quad \text { in } \mathbb{R}
$$

we can take the inverse Fourier transform of both sides of equation (5.1) to find

$$
m(-i \xi) \tilde{X}(\xi)=-\tilde{F}(\xi)+\tilde{W}(\xi) \quad \text { in } \mathbb{R}
$$

This, for almost all $\xi \in \mathbb{R}$, can be rewritten into

$$
m \frac{d\left[e^{-i t \xi} \tilde{X}(\xi)\right]}{d t}=-e^{-i t \xi} \tilde{F}(\xi)+e^{-i t \xi} \tilde{W}(\xi) \quad \text { in } \mathbb{R} .
$$

Equation (2.3) represents the model for the hard sphere of radius $r$ and mass $m$ vibrating with frequency $\xi$ and velocity $e^{-i t \xi} \bar{X}(\xi)$, subject to a fluctuating force $e^{-i t \xi} \bar{W}(\xi)$ and a drag force $e^{-i t \xi} \tilde{F}(\xi)$ at time $t$.

By solving a linearized Navier-Stokes equation subject to incompressibility and stick boundary conditions in hydrodynamics, Stokes ([42]) showed that the drag force in equation $(2.3)$ is given by

$$
\begin{aligned}
e^{-i t \xi \tilde{F}}(\xi)=6 & \pi r \eta\left\{1+r\left(\frac{\xi \rho}{2 \eta}\right)^{1 / 2}\right\} e^{-i t \xi} \tilde{X}(\xi) \\
& +3 \pi r^{2}\left(\frac{2 \rho \eta}{\xi}\right)^{1 / 2}\left\{1+\frac{2 r}{9}\left(\frac{\xi \rho}{2 \eta}\right)^{1 / 2}\right\} \frac{d\left\{e^{-i t \xi} \tilde{X}(\xi)\right\}}{d t}
\end{aligned}
$$

and so

$$
\tilde{F}(\xi)=\left\{6 \pi r \eta+6 \pi r^{2} \sqrt{\frac{\rho \eta \xi}{2}}+\left(3 \pi r^{2} \sqrt{\frac{2 \rho \eta}{\xi}}+\frac{2 \pi r^{3}}{3} \rho\right)(-i \xi)\right\} \tilde{X}(\xi)
$$

Moreover, Boussinesq ([3]) took Fourier transform of both sides of equation (2.4) to obtain the drag force $F(t)$ in equation (2.1):

$$
F(t)=2 \pi r^{3} \rho\left\{\frac{1}{3} \frac{d X(t)}{d t}+\frac{3 \eta}{r^{2} \rho} X(t)+\frac{3}{r}\left(\frac{\eta}{\pi \rho}\right)^{1 / 2} \int_{-\infty}^{t} \frac{1}{\sqrt{t-s}} \frac{d X(s)}{d s} d s\right\}
$$

Hence, equation (2.1) becomes

$$
m^{*} \dot{X}(t)=-6 \pi r \eta X(t)-6 \pi r^{2}\left(\frac{\rho \eta}{\pi}\right)^{1 / 2} \int_{-\infty}^{t} \frac{1}{\sqrt{t-s}} \dot{X}(s) d s+W(t)
$$


where $m^{*}$ is the effective mass given by

$$
m^{*}=m+\frac{2}{3} \pi r^{3} \rho
$$

This equation (2.6) is nothing but Stokes-Boussinesq-Langevin equation([44],[15],[28]). From the point of view of the theory of stochastic processes, we shall in the sequel consider a mathematical justification for the term of singular integral in the right-hand side for equation (2.6) and a mathematical meaning of its solution $X(t)$.

Substituting (2.4) into (2.2) gives us

$$
\tilde{X}(\xi)=\left\{\sqrt{2 \pi} h_{S B}(\xi)\right\} \tilde{W}(\xi)
$$

which is a relation representating equation (2.6) in the frequency world. Here $h_{S B}$ is the frequency response function on $(\mathbb{R}-\{0\}) \cup C^{+}$defined by

$$
h_{S B}(\zeta)=\frac{1}{\sqrt{2 \pi}} \frac{1}{6 \pi r \eta+m^{*}(-i \zeta)+6 \pi r^{2} \sqrt{p \eta} \sqrt{-i \zeta}}
$$

Furthermore $\sqrt{-i \zeta}$ stands for $\sqrt{-i \zeta}=\exp \frac{1}{2}(\log |\zeta|+i \operatorname{Arg}(-i \zeta))$. By using the formula

$$
\int_{0}^{\infty} \frac{1}{\lambda-i \zeta} \frac{1}{\sqrt{\lambda}} d \lambda=\pi \frac{1}{\sqrt{-i \zeta}} \quad\left(\zeta \in \mathbb{C}^{+}\right)
$$

we can rewrite the function $h_{S B}$ into

$$
h_{S B}(\zeta)=\frac{\alpha_{S B}}{\sqrt{2 \pi}} \frac{1}{\beta_{S B}-i \zeta-i \zeta \int_{0}^{\infty}(\lambda-i \zeta)^{-1} \rho_{S B}(d \lambda)}
$$

where $\alpha_{S B}$ and $\beta_{S B}$ are positive constants and $\rho_{S B}$ is a Borel measure on $[0, \infty)$ given by

$$
\left(\alpha_{S B}, \beta_{S B}, \rho_{S B}\right)=\left(\frac{1}{m^{*}}, \frac{6 \pi r \eta}{m^{*}}, \frac{6 r^{2} \sqrt{\rho \eta}}{m^{*}} \frac{1}{\sqrt{\lambda}} d \lambda\right) .
$$

It can be shown that $h_{S B}$ becomes an outer function as an $L^{2}$-function and its Fourier transform $\hat{h}_{S B}$ has the following form:

$$
\begin{aligned}
\hat{h}_{S B}(t) & =\chi_{(0, \infty)}(t) \int_{0}^{\infty} e^{-t \lambda} \nu_{S B}(d \lambda), \\
\nu_{S B}(d \lambda) & =\left(\frac{2}{\pi}\right)^{1 / 2} \alpha_{S B} e_{S B} \frac{\sqrt{\lambda}}{\left(\beta_{S B}-\lambda\right)^{2}+e_{S B}^{2} \lambda} d \lambda .
\end{aligned}
$$


Here the positive constant $e_{S B}$ is defined by

$$
e_{S B}=6 \pi r^{2} \frac{\sqrt{\rho \eta}}{m^{*}}
$$

Concerning the fluctuating force $W=(W(t) ; t \in R)$, we at first treat the white noise which is easy to be understood mathematicaly :

$$
W(t)=\alpha_{W} \dot{B}(t)
$$

where $\alpha_{W}$ is a positive constant and $\mathbf{B}=(B(t) ; t \in \mathbb{R})$ is a standard Brownian motion defined on a probability space $(\Omega, \mathcal{B}, P)$. It then follows that the stochastic process $\mathrm{X}_{\mathrm{w}}=$ $\left(X_{W}(t) ; t \in \mathbb{R}\right)$ constituted from $X(t)(t \in \mathbb{R})$ which is determined by relation (2.8) can be representated as

$$
X_{W}(t)=\frac{\alpha_{W}}{\sqrt{2 \pi}} \int_{\mathbb{R}} \hat{h}_{S B}(t-u) d B(u) .
$$

We then find that $\mathbf{X}_{\mathbf{W}}$ has the weak stationarity:

$$
\int_{\Omega} X(t) X(s) d P=R_{W}(t-s)
$$

Moreover we see from $(2.13),(2.14)$ and (2.18) that the correlation function $R_{W}$ of the process $\mathbf{X}_{\mathrm{w}}$ can be calculated as follows:

$$
\begin{gathered}
R_{W}(t)=\frac{\alpha_{W}^{2}}{2 \pi} \int_{\mathbb{R}} \hat{h}_{S B}(t+u) \hat{h}_{S B}(u) d u=\alpha_{W}^{2} \int_{0}^{\infty} e^{-|t| \lambda} \sigma_{S B}(d \lambda), \\
\sigma_{S B}(d \lambda)=\frac{\alpha_{S B}^{2}}{\pi} \frac{e_{S B}}{\beta_{S B}+\lambda+e_{S B} \sqrt{\lambda}} \frac{\sqrt{\lambda}}{\left(\beta_{S B}-\lambda\right)^{2}+e_{S B}^{2} \lambda} d \lambda
\end{gathered}
$$

We now shall consider the case where the fluctuating force $W=(W(t) ; t \in \mathbb{R})$ is $\mathrm{Kubo}$ noise $I=(I(\varphi) ; \varphi \in \mathcal{S}(\mathbb{R}))$. It follows from Kubo's linear response theory ([13],[14]) that the random force $I$ to be wanted and the weakly stationary process $\mathrm{X}_{\mathrm{K}}=\left(X_{K}(t) ; t \in \mathbb{R}\right)$ which is a solution of equation (2.6) replaced $W$ by $I$ are characterized by the following relation:

$$
\int_{\mathbb{R}} X_{K}(u) \varphi(u) d u=\frac{1}{\sqrt{2 \pi}} \int_{0}^{\infty} R_{K}(u) I(\varphi(\cdot+u)) d u \quad[\varphi \in \mathcal{S}(\mathbb{R})] .
$$


Here $R_{K}$ is the correlation function of $\mathrm{X}_{\mathrm{K}}$ :

$$
R_{K}(t-s) \equiv \int_{\Omega} X_{K}(t)(\omega) X_{K}(s)(\omega) d P(\omega)
$$

By applying the inverse Fourier transform to both sides of equation (2.21), we see that it is necessary for the following relation to hold in order to arrive at (2.8) replaced $W$ by $I$ :

$$
R_{K}(t)=\hat{h}_{S B}(|t|)=\int_{0}^{\infty} e^{-|t| \lambda} \nu_{S B}(d \lambda)
$$

We shall take the inverse argument. Since the function in right-hand side of (2.23) is always non-negative deifinite, there exists a weakly stationary process $\mathbf{X}_{\mathrm{K}}=\left(X_{K}(t) ; t \in \mathbb{R}\right)$ with the function $R_{K}$ as its correlation function. Further we find that the stochastic process determined by relation (2.8) is nothing but the stationary random distribution $\mathrm{I}=(I(\varphi) ; \varphi \in \mathcal{S}(\mathbb{R}))$ to be called Kubo noise.

This is the prescription that we can construct the stationary solution $\mathbf{X}_{\mathbf{K}}$ and $\mathrm{Kubo}$ noise I from our equation (2.6) (三 model), which is the essential part of Kubo's linear response theory. We had a good deal of labor to establish this prescription ([27],[28]). Really, the research to deduce a mathematical structure of Kubo's fluctuation-dissipation theorem in statistical physics entirely depended upon the introduction of Kubo noise, because there was no notion of Kubo noise in Kubo's linear response theory.

We might treat other noises besides the white noise and $\mathrm{Kubo}$ noise as random forces in equation (2.6). The way we can take according to FDP stated in $\S 1$ is to answer the following questions:

$$
\left\{\begin{array}{l}
\text { What kinds of qualitative nature does the solution }\left(\mathrm{X}_{\mathrm{W}}, \mathrm{X}_{\mathrm{K}}\right) \\
\text { of equation (2.6) have? }
\end{array}\right.
$$

$$
\left\{\begin{array}{l}
\text { What kinds of equations can govern a stochastic process } \\
\text { with such a qualitative nature? }
\end{array}\right.
$$

By (2.19) and (2.23), we can give the question (*) an answer that both $\mathrm{X}_{\mathrm{W}}$ and $\mathrm{X}_{\mathrm{K}}$ have $T$-positivity. Concerning its definition and question (**), we shall discuss again in the sequel. 


\section{§3. T-positivity and KMO-Langevin equation (continuous system)}

Let $\mathbf{X}=(X(t) ; t \in R)$ be a real weakly stationary process with expectation 0 whose correlation function $R$ has the following representation to be called $T$-positivity:

$$
R(t)=\int_{[0, \infty)} e^{-|t| \lambda} \sigma(d \lambda) \quad(t \in \mathbb{R}) .
$$

Here we put the integrability condition on the Borel measure $\sigma$ on $[0, \infty)$ :

$$
\begin{aligned}
& \sigma(\{0\})=0, \quad 0<\sigma([0, \infty))<\infty, \\
& \int_{0}^{\infty} \lambda^{-1} \sigma(d \lambda)<\infty, \\
& \int_{0}^{\infty} \lambda \sigma(d \lambda)<\infty .
\end{aligned}
$$

We denote by $\Delta=\Delta(\xi)(\xi \in \mathbb{R})$ the spectral density of the correlation function $R$ :

$$
R(t)=\int_{\mathbb{R}} e^{-i t \xi} \Delta(\xi) d \xi \quad(t \in \mathbb{R}) .
$$

It then follows from (3.1) that

$$
\Delta(\xi)=\frac{1}{\pi} \int_{0}^{\infty} \frac{\lambda}{\lambda^{2}+\xi^{2}} \sigma(d \lambda) \quad(\xi \in \mathbb{R}) .
$$

Moreover let $h$ and $[R]$ be the outer function and the complex mobility function of $\mathbf{X}$, respectively:

$$
\begin{aligned}
h(\zeta) & =\exp \left(\frac{1}{2 \pi i} \int_{\mathbb{R}} \frac{1+\lambda \zeta}{\lambda-\zeta} \frac{\log \Delta(\lambda)}{1+\lambda^{2}} d \lambda\right) & & \left(\zeta \in \mathbb{C}^{+}\right), \\
{[R](\zeta) } & =\frac{1}{2 \pi} \int_{0}^{\infty} e^{i \zeta t} R(t) d t & & \left(\zeta \in \mathbb{C}^{+}\right) .
\end{aligned}
$$

By approximating the Borel measure $\sigma$ by a sequence $\left(\sigma_{n} ; n \in \mathbb{N}\right)$ of Borel measures satisfying the following conditon (3.9) together with (3.2) and (3.3):

$$
\int_{0}^{\infty} \lambda^{2} \sigma(d \lambda)<\infty
$$

which is the one treated in [21]-[24] stronger than (3.4), we can apply the results in [21] to obtain the representation theorem of the outer function $h$ and the complex mobility function $[R]$. 
Theorem 3.1 ([27]). There exist uniquely two triplets $\left(\alpha_{j}, \beta_{j}, \rho_{j}\right)(j=1,2)$ satisfying the following properties:

(i) $\alpha_{j}>0, \beta_{j}>0$,

(ii) $\rho_{j}$ is a Borel measure on $[0, \infty)$ with $\rho_{j}(\{0\})=0$ and $\int_{0}^{\infty} \frac{1}{\lambda+1} \rho_{j}(d \lambda)<\infty$,

(iii) $\quad h(\zeta)=\frac{\alpha_{1}}{\sqrt{2 x}} \frac{1}{\beta_{1}-i \zeta-i \zeta \int_{0}^{\infty}(\lambda-i \zeta)^{-1} \rho_{1}(d \lambda)} \quad\left(\zeta \in C^{+}\right)$,

(iv) $[R](\zeta)=\frac{\alpha_{2}}{\sqrt{2 \pi}} \frac{1}{\beta_{2}-i \zeta-i \zeta \int_{0}^{\infty}(\lambda-i \zeta)^{-1} \rho_{2}(d \lambda)} \quad\left(\zeta \in \mathbb{C}^{+}\right)$.

Defintion 3.1. We shall call the triplet $\left(\alpha_{1}, \beta_{1}, \rho_{1}\right)\left(\right.$ resp. $\left.\left(\alpha_{2}, \beta_{2}, \rho_{2}\right)\right)$ the first (resp. the second) KMO-Langevin data associated with the correlation function $R$

Remark 3.1. The integrability condition (3.4) is not necessary for the introduction of the second KMO-Langevin data $\left(\alpha_{2}, \beta_{2}, \rho_{2}\right)$.

For the purpose of substantiating this nomenclature, we shall derive a stochastic differential equation describing the time evolution of $\mathbf{X}$, which will simultaneously answer the problem $(* *)$ stated in the last part of $\$ 2$.

We find from Karhunen's representation theorem ([5]) in the spectral theory for the weakly stationary process $\mathbf{X}$ that there exists an orthogonal process $\mathbf{B}=(B(t) ; t \in \mathbb{R})$ satisfying the following: for any $s, t \in \mathbb{R}$,

$$
\begin{gathered}
\int_{\Omega} B(s) B(t) d P=s \wedge t \\
X(t)=\frac{1}{\sqrt{2 \pi}} \int_{-\infty}^{t} \hat{h}(t-u) d B(u)
\end{gathered}
$$

$$
\text { the linear hull of }\{X(u) ; u \leq t\}=\text { the linear hull of }\{B(u)-B(v) ; u, v \leq t\} \text {. }
$$

The random stationary distribution $\dot{\mathbf{B}}$ coming from the differentiation of $\mathbf{B}$ is called the standard white noise. On the other hand, Kubo noise $I=(I(\varphi) ; \varphi \in \mathcal{S}(\mathbb{R}))$ is the random stationary distribution defined by

$$
I(\varphi)=\int_{\mathbb{R}}\left(\frac{h}{[R]} \hat{\varphi}\right)^{\sim}(s) d B(s) .
$$


Coresponding to (3.11) and (3.12), we have the following: for any $t \in \mathbb{R}, \varphi \in \mathcal{S}(\mathbb{R})$,

$$
\int_{\mathbb{B}} X(u) \varphi(u) d u=\frac{1}{\sqrt{2 \pi}} \int_{0}^{\infty} R(u) I(\varphi(\cdot+u)) d u
$$

(3.15) the linear hull of $\{X(u) ; u \leq t\}=$ the linear hull of $\{I(\varphi)$; supp $\varphi \subset(-\infty, t]\}$.

We put $\varphi=\delta(\cdot-t)$ to obtain an intutive representation for (3.13) and (3.14). We then find that (3.13) can be rewritten into

$$
I(t)=\frac{1}{2 \pi} \int_{-\infty}^{t}\left(\frac{h}{[R]}\right)^{\wedge}(t-u) d B(u)
$$

and (3.14) has the following representation analogous to (3.11):

$$
X(t)=\frac{1}{\sqrt{2 \pi}} \int_{-\infty}^{t} R(t-u) I(u) d u
$$

We have derived the standard white noise $\dot{\mathbf{B}}$ and Kubo noise $\mathbf{I}$ as random forces associated with the given weakly stationary process $\mathbf{X}$. The representation (3.11) (resp. (3.14)') can be regarded as a construction of a model such that $\mathbf{X}$ is the common output, $\dot{\mathbf{B}}$ (resp. I) is the input and $\hat{h}$ (resp. $\chi[0, \infty) R$ ) is the response function. Following the idea in FDP, we find that these models can be rewritten into the following equations.

Theorem 3.2 ([27]). As random distributions, $\mathrm{X}$ and $\dot{\mathrm{B}}$ (resp. $\mathrm{X}$ and $\mathrm{I}$ ) satisfy the following stochastic differential equation (3.16) (resp. (3.17)):

$$
\begin{aligned}
& \dot{\mathbf{X}}=-\beta_{1} \mathrm{X}-\lim _{\epsilon \downarrow 0} \gamma_{1, \varepsilon} * \dot{\mathrm{X}}+\alpha_{1} \dot{\mathrm{B}} \\
& \dot{\mathrm{X}}=-\beta_{2} \mathrm{X}-\lim _{\epsilon \downarrow 0} \gamma_{2, \varepsilon} * \dot{\mathrm{X}}+\alpha_{2} \mathrm{I}
\end{aligned}
$$

Here $\gamma_{j, e}(j=1,2)$ are the functions given by

$$
\gamma_{j, \varepsilon}(t)=\chi(0, \infty)(t) \int_{c}^{\infty} e^{-t \lambda} \rho_{j}(d \lambda)
$$

Defintion 3.2. We shall call the stochastic differential equation (3.16) (resp. (3.17)) the first (resp. the second) KMO-Langevin equation associated with the weakly stationary process $\mathbf{X}$. 
Example 3.1. For each $v>0, \beta>0$, let $\mathrm{X}_{v, \beta}$ be a weakly stationary process with the correlation function $R_{v, \beta}$ of the form

$$
R_{v, \beta}(t)=v e^{-\beta|t|} \quad(t \in \mathbb{R})
$$

This is the case where $\sigma$ in (3.1) is a point measure $\sigma=v \delta_{\beta}$. The process $\mathbf{X}_{v, \beta}$ is called Ornstein-Uhlenbeck's Brownian motion which was investigated in the study of Einstein and Langevin. It follows that the outer function $h_{v, \beta}$ and the complex mobility function $\left[R_{v, \beta}\right]$ are given by the following:

$$
h_{v, \beta}(\zeta)=\frac{\sqrt{2 v \beta}}{2 \pi} \frac{1}{\beta-i \zeta} \quad \text { and } \quad\left[R_{v, \beta}\right](\zeta)=\frac{v}{2 \pi} \frac{1}{\beta-i \zeta} \quad\left(\zeta \in \mathbb{C}^{+}\right) .
$$

Hence the first and the second KMO-Langevin data associated with the correlation $R_{v, \beta}$ become

$$
\left(\alpha_{1}, \beta_{1}, \rho_{1}\right)=(\sqrt{2 v \beta}, \beta, 0) \text { and }\left(\alpha_{2}, \beta_{2}, \rho_{2}\right)=(v, \beta, 0)
$$

Furthermore $\mathbf{X}_{v, \beta}$ satisfy the following first and second KMO-Langevin equations:

$$
\begin{aligned}
& \dot{\mathbf{X}}_{v, \beta}=-\beta \mathbf{X}_{v, \beta}+\sqrt{2 v \beta} \dot{\mathbf{B}}_{v, \beta}, \\
& \dot{\mathbf{X}}_{v, \beta}=-\beta \mathbf{X}_{v, \beta}+v \mathrm{I}_{v, \beta} .
\end{aligned}
$$

Here $\dot{\mathbf{B}}_{v, \beta}$ and $\mathrm{I}_{v, \beta}$ are the standard white noise and Kubo noise associated with $\mathbf{X}_{v, \beta}$, respectively. It follwos from (3.22) and (3.23) that both random distributions are equal up to positive constants:

$$
\mathrm{I}_{v, \beta}=\sqrt{\frac{2 \beta}{v}} \dot{\mathrm{B}}_{v, \beta}
$$

§4. T-positivity and KMO-Langevin equation (discrete system)

Let $\mathrm{X}=(X(n) ; n \in \mathbb{Z})$ be a real weakly stationary process defined on a probability space $(\Omega, \mathcal{B}, P)$ with expectation 0 and correlation function $R$ of the form ( $T$-positivity):

$$
R(n)=\int_{[-1,1]} t^{|n|} \sigma(d t) \quad(n \in \mathbb{Z})
$$


Here the Borel measure $\sigma$ on $[-1,1]$ satisfies the following integrability conditions:

$$
\begin{aligned}
& \sigma(\{-1,1\})=0, \quad 0<\sigma([-1,1])<\infty, \\
& \int_{-1}^{1}\left(\frac{1}{1+t}+\frac{1}{1-t}\right) \sigma(d t)<\infty .
\end{aligned}
$$

The spectral measure $\Delta=\Delta(\theta)$ of the correlation function $R$ detemined by the relation

$$
R(n)=\int_{-\pi}^{\pi} e^{-i n \theta} \Delta(\theta) d \theta \quad(n \in \mathbb{Z})
$$

satisfies

$$
\Delta(\theta)=\frac{1}{\pi} \int_{-1}^{1} \frac{1-t^{2}}{\left|1-t e^{i \theta}\right|^{2}} \sigma(d t) \quad(\theta \in(-\pi, \pi)) .
$$

The outer function $h$ and the complex mobility function $[R]$ of the process $\mathrm{X}$ are respectively defined by

$$
\begin{aligned}
h(z) & =\exp \left(\frac{1}{4 \pi} \int_{-\pi}^{\pi} \frac{e^{i \theta}+z}{e^{i \theta}-z} \log \Delta(\theta) d \theta\right) & & (z \in \mathbb{C},|z|<1), \\
{[R](z) } & =\frac{1}{2 \pi} \sum_{n=0}^{\infty} R(n) z^{n} & & (z \in \mathbb{C},|z|<1) .
\end{aligned}
$$

By using a homeomorohism $\varphi: \varphi(t)=(1+t) /(1-t)$ from $(-1,1)$ in $[0, \infty)$, we transform the Borel measure $\sigma$ on $(-1,1)$ to the Borel measure $\sigma_{c}=\varphi(\sigma)$ on $[0, \infty)$ and apply Theorem 3.1 for the continuous case to obtain the representation theorem for the outer function $h$ and the complex mobility function $[R]$ for the discrete case:

Theorem $4.1([32],[33])$. There exist uniquely two triplets $\left(\alpha_{j}, \beta_{j}, \rho_{j}\right)(j=1,2)$ satisfying the following properties:

(i) $\alpha_{j}>0, \beta_{j}>0$,

(ii) $\rho_{j}$ is a Borel measure on $[-1,1]$ with $\rho_{j}(\{-1,1\})=0$ and $\rho_{j}([-1,1])<\infty$,

(iii) $\quad h(z)=\frac{\alpha_{1}}{\sqrt{2 \pi}} \frac{1}{\beta_{1}(1+z)+1-z+\left(1-z^{2}\right) \int_{-1}^{1}(1-t z)^{-1} \rho_{1}(d t)} \quad(z \in \mathbb{C},|z|<1)$,

(iv) $[R](z)=\frac{\alpha_{2}}{\sqrt{2 \pi}} \frac{1}{\beta_{2}(1+z)+1-z+\left(1-z^{2}\right) \int_{-1}^{1}(1-t z)^{-1} \rho_{2}(d t)} \quad(z \in \mathbb{C},|z|<1)$. 
Defintion 4.1. We shall call the triplet $\left(\alpha_{1}, \beta_{1}, \rho_{1}\right)\left(\right.$ resp. $\left.\left(\alpha_{2}, \beta_{2}, \rho_{2}\right)\right)$ the first (resp. the second) KMO-Langevin data associated with the correlation function $R$.

We shall derive a stochastic difference equation describing the time evolution of $\mathbf{X}$ in the discrete system, corresponding to the stochastic differential equations (3.16) and (3.17) in the continuous system, which will answer the discrete version to the question $(* *)$ in $\$ 2$. It follows from the spectral theory of the weakly stationary process $X$ that there exist a weakly stationary process $\xi=(\xi(n) ; n \in \mathbb{Z})$, to be called the standard white noise, satisfying the following:

$$
\begin{gathered}
\int_{\Omega} \xi(n) \xi(m) d P=\delta_{n m} \quad(n, m \in \mathbb{Z}), \\
X(n)=\frac{1}{\sqrt{2 \pi}} \sum_{m=-\infty}^{n} \hat{h}(n-m) \xi(m) \quad(n \in \mathbb{Z}), \\
\mathbf{M}_{-\infty}^{n}(\mathbf{X})=\mathbb{M}_{-\infty}^{n}(\xi) \quad(n \in \mathbb{Z}) .
\end{gathered}
$$

For an $\mathbb{R}^{d}$ valued stochastic process $Y=\left({ }^{t}\left(Y_{1}(n), \cdots, Y_{d}(n)\right) ; n \in \mathbb{Z}\right)$ defined on the probability space $(\Omega, \mathcal{B}, P)$, generally, we shall denote by $\mathbf{M}_{n}^{m}(Y)$ the closed subspace of $L^{2}(\Omega, \mathcal{B}, P)$ for $n, m,-\infty \leq n \leq m \leq \infty$ :

$$
\mathrm{M}_{n}^{m}(Y) \equiv \text { the linear hull of }\left\{Y_{j}(k) ; 1 \leq j \leq d, n \leq k \leq m\right\}
$$

Corresponding to the intuitive representation (3.13)' in the continuous system, Kubo noise $\mathrm{I}=(I(n) ; n \in \mathbb{Z})$ in the discrete system is a weakly stationary process defined by

$$
I(n)=\frac{1}{2 \pi} \sum_{m=-\infty}^{n}\left(\frac{h}{[R]}\right)^{\wedge}(n-m) \xi(m) .
$$

As the discrete version of (3.14) ((3.14)') and (3.15) in the continuous system, we have

$$
X(n)=\frac{1}{\sqrt{2 \pi}} \sum_{m=-\infty}^{n} R(n-m) I(m) \quad(n \in \mathbb{Z})
$$




$$
\dot{M}_{-\infty}^{n}(\mathrm{X})=\mathrm{M}_{-\infty}^{n}(\mathrm{I}) \quad(n \in \mathbb{Z})
$$

Similarly as in the continuous system, the standard white noise $\xi$ and Kubo noise I were derived, starting from the given weakly stationary process $\mathbf{X}$. The model (4.9) (resp. (4.13)) in which $\mathbf{X}$ is the common output, $\xi$ (resp. I) is the input and $\hat{h}$ (resp. $\chi_{[0, \infty)} R$ ) is the response function can be described by the following stochastic difference equation (4.15) (resp. (4.16)):

Theorem 4.2 ([32],[33]). The process $\mathrm{X}$ satisfies

$$
\begin{aligned}
& X(n)-X(n-1)=-\beta_{1}(X(n)+X(n-1))-\left(\gamma_{1} * X\right)(n)+\alpha_{1} \xi(n) \\
& X(n)-X(n-1)=-\beta_{2}(X(n)+X(n-1))-\left(\gamma_{2} * X\right)(n)+\alpha_{2} I(n) .
\end{aligned}
$$

Here $\gamma_{j}(j=1,2)$ are $l^{1}(\mathbb{Z})$-functions given by

$$
\gamma_{j}=\frac{1}{2 \pi}\left(\left(1-e^{2 i}\right) \int_{-1}^{1} \frac{1}{1-t e^{i .}} \rho_{j}(d t)\right)^{\wedge} .
$$

Defintion 4.2. We shall call the stochastic difference equation (4.15) (resp. (4.16)) the first (resp. the second) KMO-Langevin equation associated with the weakly stationary process $\mathbf{X}$.

The functions $\gamma_{j}(j=1,2)$ determined by $(4.17)$ can be representated into

$$
\gamma_{j}(n)= \begin{cases}0 & (n=-1,-2, \cdots) \\ \int_{-1}^{1} t^{n} \rho_{j}(d t) & (n=0,1) \\ \int_{-1}^{1}\left(t^{n}-t^{n-2}\right) \rho_{j}(d t) & (n=2,3, \cdots)\end{cases}
$$

and so

$$
\sum_{n=0}^{\infty} \gamma_{j}(n)=0 \text { and } \sum_{n=0}^{\infty}(-1)^{n} \gamma_{j}(n)=0
$$

Inoue ([11]) noticed the following fact: the first (resp. the second) KMO-Langevin equation (4.15) (resp. (4.16)) can be rewritten into the one of the same form as the delay 
term in the first (resp. the second) KMO-Langevin equation (3.16) (resp. (3.17)). Defining the functions $\gamma_{j, 0}(j=1,2)$ by

$$
\gamma_{j, 0}(n)= \begin{cases}0 & (n=-1,-2, \cdots) \\ \rho_{j}([-1,1]) & (n=0) \\ \int_{-1}^{1}\left(t^{n}+t^{n-1}\right) \rho_{j}(d t) & (n=1,2, \cdots),\end{cases}
$$

we have

$$
\gamma_{j, 0}(n)-\gamma_{j, 0}(n-1)=\gamma_{j}(n) \quad(n \in \mathbb{Z})
$$

This can allow us to rewrite the delay term $\left(\gamma_{j} * X\right)(n)$ in (4.15) and (4.16) into

$$
\left(\gamma_{j} * X\right)(n)=\underset{\varepsilon i \mathrm{i} . \mathrm{m} .}{n-1} \sum_{m=-\infty}^{e} \gamma_{j, 0}^{e}(n-m) \Delta X(m)+\gamma_{j}(0) \Delta X(n)
$$

Here we put

$$
\begin{gathered}
\Delta X(n)=X(n)-X(n-1), \\
\gamma_{j, 0}^{e}(n)= \begin{cases}0 & (n=-1,-2, \cdots) \\
\rho_{j}([-1+\varepsilon, 1-\varepsilon]) & (n=0) \\
\int_{-1+\varepsilon}^{1-\varepsilon}\left(t^{n}+t^{n-1}\right) \rho_{j}(d t) & (n=1,2, \cdots) .\end{cases}
\end{gathered}
$$

Therefore, we can set for each $j=1,2$

$$
\left(\alpha_{m, j}, \beta_{m, j}, \gamma_{m, j}\right)=\left(\frac{\alpha_{j}}{1+\gamma_{j}(0)}, \frac{2 \beta_{j}}{1+\gamma_{j}(0)}, \frac{\gamma_{j, 0}}{1+\gamma_{j}(0)}\right)
$$

to rewrite equation (4.15) (resp. (4.16)) into the following equation (4.26) (resp. (4.27)):

$$
\begin{aligned}
& \Delta X(n)=-\beta_{m, 1} \frac{(X(n)+X(n-1))}{2} \\
& -\underset{c \downarrow j}{\operatorname{igm}} \sum_{m=-\infty}^{n-1} \gamma_{1,0}^{\varepsilon}(n-m) \Delta X(m)+\alpha_{m, 1} \xi(n), \\
& \Delta X(n)=-\beta_{m, 2} \frac{(X(n)+X(n-1))}{2} \\
& -\underset{c \downarrow 0}{\operatorname{i} . \mathrm{m}_{0}} \sum_{m=-\infty}^{n-1} \gamma_{2,0}^{e}(n-m) \Delta X(m)+\alpha_{m, 2} I(n) \text {. }
\end{aligned}
$$


Defintion 4.3. We shall call the stochastic differential equation (4.26) (resp. (4.27)) the modified first (resp. the modified second) KMO-Langevin equation associated with the weakly stationary process $\mathrm{X}$. The triple $\left(\alpha_{m, 1}, \beta_{m, 1}, \gamma_{m, 1}\right)\left(\operatorname{resp} .\left(\alpha_{m, 2}, \beta_{m, 2}, \gamma_{m, 2}\right)\right)$ is called the modified first (resp. the modified second) KMO-Langevin data associated with the correlation function $R$.

Example 4.1. For each $v>0, p \in(-1,1)$, let $\mathrm{X}_{v, p}$ be a weakly stationary process with the correlation function $R_{v, p}$ of the form

$$
R_{v, p}(n)=v p^{|n|} \quad(n \in \mathbb{Z})
$$

This is the case where $\sigma$ in (4.1) is a point measure $\sigma=v \delta_{p}$. The process $\mathrm{X}_{v, p}$ has Markovian property. The outer function $h_{v, p}$ and the complex mobility function $\left[R_{v, p}\right]$ can be calculated as follows:

$$
h_{v, p}(z)=\sqrt{\frac{v}{2 \pi}} \frac{1-p^{2}}{1-p z} \quad \text { and } \quad\left[R_{v, p}\right](z)=\frac{v}{2 \pi} \frac{1}{1-p z} \quad(z \in \mathbb{C},|z|<1) .
$$

Hence the first and second KMO-Langevin data associated with the correlation $R_{v, p}$ become

$$
\left\{\begin{array}{l}
\left(\alpha_{v, p}^{1}, \beta_{v, p}^{1}, \rho_{v, p}^{1}\right)=\left(2 \sqrt{v \frac{1-p}{1+p}}, \frac{1-p}{1+p}, 0\right) ; \\
\left(\alpha_{v, p}^{2}, \beta_{v, p}^{2}, \rho_{v, p}^{2}\right)=\left(\sqrt{\frac{2}{\pi}} \frac{v}{1+p}, \frac{1-p}{1+p}, 0\right) .
\end{array}\right.
$$

Furthermore there exists the following relation between the standard white noise $\xi_{v, p}=$ $\left(\xi_{v, p}(n) ; n \in \mathbb{Z}\right)$ and $\mathrm{Kubo}$ noise $\mathrm{I}_{v, p}=\left(I_{v, p}(n) ; n \in \mathbb{Z}\right)$ associated with $\mathrm{X}_{v, p}$ :

$$
I_{v, p}(n)=\sqrt{2 \pi(1-p)(1+p)} \xi_{v, p}(n)
$$

\section{$\S$ 5. Alder-Wainwright effect}

[5-1] Let $\mathrm{X}_{W}$ (resp. $\mathrm{X}_{K}$ ) be the stationary solution of Stokes-Boussinesq-Langevin equation (2.6) in which the random force $W$ is the white noise $\alpha_{W} \dot{\mathbf{B}}$ (resp. Kubo noise I). It follows from the representation (2.19) and (2.23) of their correlation functions $R_{W}$ and $R_{K}$ that both the process $\mathbf{X}_{W}$ and the process $\mathbf{X}_{K}$ have $T$-positivity. Furthermore we have the following Alder-Wainwright effect $([43],[15],[28])$ : 
Alder-Wainwright effect.

$$
\begin{aligned}
& \lim _{t \rightarrow \infty}\left(\beta_{S B} t\right)^{3 / 2} R_{W}(t) \\
& =\frac{\sqrt{\pi} R_{W}(0)}{2}\left\{\int_{0}^{\infty} \frac{\sqrt{y}}{(1+y+a \sqrt{y})\left((1-y)^{2}+a^{2} y\right)} d y\right\}^{-1}, \\
& \lim _{t \rightarrow \infty}\left(\beta_{S B} t\right)^{3 / 2} R_{K}(t)=\frac{R_{K}(0)}{2 \sqrt{\pi}} a .
\end{aligned}
$$

Here it is known ([15]) that the constant

$$
a=\left(\frac{6 \pi \gamma \rho^{3}}{m^{*}}\right)^{1 / 2}
$$

has the following physical meaning:

$$
a=3\left(1+2 \frac{\rho_{0}}{\rho}\right)^{-1 / 2}
$$

where $\rho_{0}$ and $\rho$ are respectively the density of the hard sphere and the liquid in the same situation as in the derivation of equation (2.6).

[5.2] We shall show that a mathematical background of Alder-Wainwright effect (5.1) and (5.2) for Stokes-Boussinesq-Langevin equation (2.6) lies in a long time behavior of the integral kernel $\sqrt{t}^{-1}$ in the delay term. Let $p$ be a positive constant and $L$ a slowly varying function at infinity:

$$
\lim _{t \rightarrow \infty} \frac{L(s t)}{L(t)}=1 \quad \text { for any } s>0 .
$$

We denote by $R$ the correlation function of the common stationary solution $\mathbf{X}$ for both the first and the second KMO-Langevin equations (3.16) and (3.17). We then find that Alder-Wainwright effect can be clarified as a polynomial decay for $R$ as follows:

Theorem 5.1 ([31],[10]). The following (i) (iii) are equivalent:

$$
\begin{array}{lll}
R(t) \sim t^{-(1+p)} L(t) & \text { as } t \rightarrow \infty . \\
\gamma_{1}(t) \sim \alpha_{1}^{-2} \beta_{1}^{3} p t^{-p} L(t) & \text { as } t \rightarrow \infty . \\
\gamma_{2}(t) \sim \sqrt{2 \pi}^{-1} \alpha_{2}^{-1} \beta_{2}^{2} p t^{-p} L(t) & \text { as } t \rightarrow \infty
\end{array}
$$


[5.3] Under the following condition

$$
\rho_{j}([-1,0))=0 \quad(j=1,2)
$$

Inoue ([11]) showed Alder-Wainwright effect for both the modified first and the modified second KMO-Langevin equations (4.26) and (4.27) in the discrete system.

Theorem 5.2 ([11]). The following (i) (iii) are equivalent:

$$
\begin{aligned}
R(n) & \sim n^{-(1+p)} L(n) & & \text { as } n \rightarrow \infty . \\
\gamma_{m, 1}(n) & \sim \alpha_{m, 1}^{-2} \beta_{m, 1}^{3} p n^{-p} L(n) & & \text { as } n \rightarrow \infty . \\
\gamma_{m, 2}(n) & \sim \sqrt{2 \pi}^{-1} \alpha_{m, 2}^{-1} \beta_{m, 2}^{2} p n^{-p} L(n) & & \text { as } n \rightarrow \infty .
\end{aligned}
$$

Remark 5.1. The condition (5.6) does not necessarily imply the following:

$$
\sigma_{j}([-1,0))=0 \quad(j=1,2) .
$$

Remark 5.2. We are succeeded in characterizing an exponential decay of the correlation function $R$ as the one of the delay functions $\gamma_{j}(j=1,2)$ in KMO-Langevin equations ([37]). Remark 5.3. The case where conditions (3.3) and (4.3) do not hold, that is, the diffusion constant in (6.1) and (6.5) of the next section is divergent interests us in relation to certain critical phenomena of random system and is now under investigation.

\section{§ 6. Fluctuation-Dissipation Theorem}

The heart in fluctuation-dissipation theorem (FDT) in FDP stated in $§ 1$ lies in a philosophical understanding that any phenomena with a complex behavior, under the stationary situation, its equation of motion describing the time evolution can be separated into a random chaotic part (fluctuant term) and a dynamical calm part (dissipative term) and certain relation holds between both terms.

It seems that in the course of the action of one's research, the process of study with trouble and joy corresponds with the fluctuant part and the action of the presentation of the results of study corresponds with the dissipative part. We shall suffer some severe 
criticisms for the presentation of our unexpected results, but we shall put them on ourselves as the fluctuating force for our research and present some results of study equal to our endeavour after further action of study. It seems that the situation where we can repeatedly continue to study is stationary.

[6.1] We shall now examine how the philosophical understanding of FDT stated above can be mathematically representated. For the weakly stationary process $\mathrm{X}$ treated in $\S 3$, we define its diffusion constant $D$ by

$$
D \equiv \lim _{t \rightarrow \infty} \frac{\int_{\Omega}\left(\int_{0}^{t} X(s) d s\right)^{2} d P}{2 t}
$$

which can be calculated as follows:

$$
D=\int_{0}^{\infty} R(t) d t=\int_{0}^{\infty} \frac{1}{\lambda} \sigma(d \lambda) .
$$

We shall at first consider Ornstein-Uhlenbeck's Brownian motion $\mathbf{X}_{v, \beta}$ treated in Example 3.1 and explain FDT on the basis of the first KMO-Langevin equation (3.22). Denoting by $D_{v, \beta}$ the diffusion constant of $\mathrm{X}_{v, \beta}$, we have the following relations:

$$
\begin{aligned}
\frac{1}{\beta_{1}-i \zeta} & =\frac{1}{R_{v, \beta}(0)} \frac{h_{v, \beta}(\zeta)}{\int_{\mathbb{R}} \operatorname{Re} h_{v, \beta}(\xi+i 0) d \xi} \\
\frac{\alpha_{1}^{2}}{2} & =R_{v, \beta}(0) \beta_{1} \\
D_{v, \beta} & =\frac{R_{v, \beta}(0)}{\beta_{1}} \\
D_{v, \beta} & =\frac{\alpha_{1}^{2}}{2 \beta_{1}^{2}}
\end{aligned}
$$

We shall observe in turn how these relations can be extented for a general weakly stationary process $\mathrm{X}$. Concerning relation $(\mathrm{A})$, we have

A generalized FDT of the first kind. ([28]). On the basis of the first and second KMO-Langevin equations (3.16) and (3.17), the following hold on $\mathbb{C}^{+} \cup(\mathbb{R}-\{0\})$, respectively:

$$
\frac{1}{\beta_{1}-i \zeta-i \zeta \lim _{e \downarrow 10} \int_{0}^{\infty} e^{i \zeta t} \gamma_{1, \varepsilon}(t) d t}=\frac{h(\zeta)}{\int_{\mathbb{R}} \operatorname{Reh}(\xi+i 0) d \xi}
$$




$$
\frac{1}{\beta_{2}-i \zeta-i \zeta \lim _{\epsilon \downarrow 0} \int_{0}^{\infty} e^{i \zeta t} \gamma_{2, c}(t) d t}=\frac{1}{R(0)} \int_{0}^{\infty} e^{i \zeta t} R(t) d t
$$

Concerning relation $(B)$, we have

A generalized FDT of the second kind. ([28]).

$$
\frac{\alpha_{1}^{2}}{2}=R(0) C_{\beta_{1}, \gamma_{1}}
$$

$$
\Delta_{\alpha_{2} \mathrm{I}}(d \xi)=\frac{R(0)}{\pi}\left\{R e\left(\beta_{2}-i \xi \lim _{\epsilon \downarrow 0} \int_{0}^{\infty} e^{i \xi t} \gamma_{2, e}(t) d t\right)\right\} d \xi .
$$

Here $C_{\beta_{1}, \gamma_{1}}$ is a constant defined by

$$
C_{\beta_{1}, \gamma_{1}}=\pi\left\{\int_{\mathbb{R}}\left|\beta_{1}+(-i \xi)\left(1+\lim _{e \downarrow 0} \int_{0}^{\infty} e^{i \xi t} \gamma_{1, \varepsilon}(t) d t\right)\right|^{-2} d \xi\right\}^{-1}
$$

and $\Delta_{\alpha_{2} \mathrm{I}}$ stands for the spectral measure of $\alpha_{2} \mathrm{I}$.

We shall turn to Einstein's relation (C).

A generalized Einstein's relation (1). ([28]). On the basis of the first and second KMO-Langevin equations (3.16) and (3.17), we have

$$
\begin{gathered}
D=\frac{R(0)}{\beta_{1}} \frac{C_{\beta_{1}, \gamma_{1}}}{\beta_{1}}, \\
D=\frac{R(0)}{\beta_{2}} .
\end{gathered}
$$

Moreover we see that

$$
\frac{C_{\beta_{1}, \gamma_{1}}}{\beta_{1}} \geq 1
$$

and the equality in (6.4) holds only for the case where $\mathrm{X}$ is Ornstein-Uhlenbeck's Brownian motion.

Hence, a classical Einstein's relation (C) always holds on the basis of the second KMOLangevin equation (3.17), but deviates according to the degree of the positive constant $C_{\beta_{1, \gamma_{1}}} / \beta_{1}-1$ on the basis of the first KMO-Langevin equation (3.16).

However, the affairs are in reverse order about relation (D). 
A generalized Einstein's relation (2). ([28]). The following relation

$$
D=\frac{\alpha_{1}^{2}}{2 \beta_{1}^{2}}
$$

always holds on the basis of the first KMO-Langevin equation (3.16). But, this does not hold on the basis of the second KMO-Langevin equation (3.17).

[6.2] We shall observe in the sequel what kinds of FDT the weakly stationary process $\mathbf{X}$ with discrete time has. FDT has never been established in irreversible statistical physics of discrete system. We define a diffusion constant $D$ associated with $\mathrm{X}$ by

$$
D \equiv \lim _{N \rightarrow \infty} \frac{1}{2 N} \int_{\Omega}\left(\sum_{n=0}^{N} X(n)\right)^{2} d P=\sum_{n=0}^{\infty} R(n)-\frac{R(0)}{2} \text {. }
$$

A generalized FDT of the first kind. ([32],[33]). On the basis of the first and second KMO-Langevin equations (4.15) and (4.16), we have the following

$$
\frac{1}{\beta_{1}\left(1+e^{i \theta}\right)+1-e^{i \theta}+2 \pi \tilde{\gamma}_{1}(\theta)}=\frac{h\left(e^{i \theta}\right)}{2 \lim _{\tau \downarrow-\pi} h\left(e^{i \tau}\right)} \quad(\theta \in(-\pi, \pi)),
$$

$$
\frac{1}{\beta_{2}\left(1+e^{i \theta}\right)+1-e^{i \theta}+2 \pi \tilde{\gamma}_{2}(\theta)}=\frac{[R]\left(e^{i \theta}\right)}{2 \lim _{\tau \downarrow-\pi}[R]\left(e^{i \tau}\right)}(\theta \in(-\pi, \pi)) .
$$

A generalized FDT of the second kind. ([32],[33]). On the basis of the first and second KMO-Langevin equations (4.15) and (4.16), we have the following

$$
\frac{\alpha_{1}^{2}}{2}=R(0) C_{\beta_{1}, \gamma_{1}}
$$

$$
\begin{array}{r}
\Delta_{\alpha_{2} I}(d \theta)=\frac{R(0)}{2 \pi}\left\{2\left(1+\beta_{2}+\gamma_{2}(0)\right) \operatorname{Re}\left(\beta_{2}\left(1+e^{i \theta}\right)+1-e^{i \theta}+2 \pi \tilde{\gamma}_{2}(\theta)\right)\right. \\
\left.-\left|\beta_{2}\left(1+e^{i \theta}\right)+1-e^{i \theta}+2 \pi \tilde{\gamma}_{2}(\theta)\right|^{2}\right\} d \theta
\end{array}
$$

Here $C_{\beta_{1}, \gamma_{1}}$ is a constant defined by

$$
C_{\beta_{1}, \gamma_{1}}=\pi\left\{\int_{-\pi}^{\pi}\left|\beta_{1}\left(1+e^{i \theta}\right)+1-e^{i \theta}+2 \pi \tilde{\gamma}_{1}(\theta)\right|^{-2} d \theta\right\}^{-1}
$$


and $\Delta_{\alpha_{2} I}$ stands for the spectral measure of $\alpha_{2} I$.

A generalized Einstein's relation (1). ([32],[33]). On the basis of the first (resp. the second and the modified second) KMO-Langevin equations (4.15) (resp. (4.16) and (4.27)), the following relations hold, respectively:

$$
\begin{gathered}
D=\frac{R(0)}{2 \beta_{1}} \frac{C_{\beta_{1}, \gamma_{1}}}{2 \beta_{1}}, \\
D=\frac{R(0)}{2 \beta_{2}}\left(1+\gamma_{2}(0)\right),
\end{gathered}
$$

$$
D=\frac{R(0)}{\beta_{m, 2}}
$$

Corresponding to inequality (6.4), we obtain

$$
\frac{C_{\beta_{1}, \gamma_{1}}}{2 \beta_{1}} \geq 1 .
$$

Similarly as in the continuous case, it follows that the equality in (6.7) holds only for the case $\rho_{1}=0$, that is, the weakly stationary process with Markovian property treated in Example 4.1. Therefore, we find in the discrete system that the classical Einstein's relation (C) for the continuous system deviates on the basis of both the first and the second KMOLangevin equations (4.15) and (4.16), but it is conserved on the basis of the modified second KMO-Langevin equation (4.27).

Finally we have the following corresponding to relations (D) and (D-1) for the continuous system.

A generalized Einstein's relation (2). ([32]). On the basis of the first and the modified first KMO-Langevin equations (4.15) and (4.26), we have

$$
D=\frac{\alpha_{1}^{2}}{2\left(2 \beta_{1}\right)^{2}}
$$

$(\mathrm{D}-1-\mathrm{m})$

$$
D=\frac{\alpha_{m, 1}{ }^{2}}{2\left(\beta_{m, 1}\right)^{2}}
$$


But neither the second or the modified second KMO-Langevin equations (4.16) and (4.27) have these relations.

\section{§ 7. $\mathrm{KM}_{2} \mathrm{O}$-Langevin equation and FDT}

Let $\mathrm{X}=(X(n) ;|n| \leq N)$ be an $\mathbb{R}^{d}$ valued weakly stationary process defined on a probability space $(\Omega, \mathcal{B}, P)$ with expectation vector 0 . Here $d, N$ are fixed nutural numbers and we do not assume that the process $\mathrm{X}$ has $T$-positivity. Denoting by $R$ the correlation matrix function of the process $\mathrm{X}$, we define for each $n, 1 \leq n \leq N$, a Toeplitz matrix $S_{n}(\in M(n d ; \mathbb{R}))$ by

$$
S_{n}=\left(\begin{array}{cccc}
R(0) & R(1) & \ldots & R(n-1) \\
{ }^{t} R(1) & R(0) & \ldots & R(n-2) \\
\vdots & \vdots & \ddots & \vdots \\
{ }^{t} R(n-1) & { }^{t} R(n-2) & \ldots & R(0)
\end{array}\right) .
$$

It is to be noted that

$$
{ }^{t} R(n)=R(-n)
$$

We remark that under the following condition

$$
R(0) \in G L(d ; \mathbb{R})
$$

either (7.4) or (7.5) holds:

$$
\begin{aligned}
& S_{n} \in G L(n d ; \mathbb{R}) \quad(1 \leq n \leq N), \\
& \begin{cases}S_{n} \in G L(n d ; \mathbb{R}) & \left(1 \leq n \leq N_{0}\right) \\
S_{n} \notin G L(n d ; \mathbb{R}) & \left(N_{0}+1 \leq n \leq N\right),\end{cases}
\end{aligned}
$$

where $N_{0}=\max \left\{n \in\{1, \cdots, N\} ; \operatorname{det}\left(S_{n}\right) \neq 0\right\}$.

By reminding of the notation (4.11), we define two $\mathbb{R}^{d}$ valued stochastic processes $\nu_{+}=\left(\nu_{+}(n) ; 0 \leq n \leq N\right)$ and $\nu_{-}=\left(\nu_{-}(-n) ; 0 \leq n \leq N\right)$ by

$$
\nu_{+}(n)=X(n)-P_{\mathbf{M}_{0}^{n-1}(\mathbf{X})} X(n) \quad \text { and } \quad \nu_{-}(-n)=X(-n)-P_{\mathbf{M}_{-n+1}^{0}}(\mathbf{X}) X(-n)
$$


where $P_{\mathbf{M}_{n}^{m}(\mathbf{X})}$ stands for the projection operator on the closed subspace $\mathbf{M}_{n}^{m}(\mathbf{X})(n \leq m)$ with the convention $\mathrm{M}_{0}^{-1}(\mathrm{X})=\mathrm{M}_{1}^{0}(\mathrm{X})=\{0\}$. Let $V_{+}(n)$ and $V_{-}(n)$ be the variance matrix of $\nu_{+}(n)$ and $\nu_{-}(-n)$, respectively. It then follows that

Causal Relation ([35],[40]). For each $n, m, 0 \leq n, m \leq N$,

$$
\begin{gathered}
\nu_{+}(0)=\nu_{-}(0)=X(0) \\
\int_{\Omega} \nu_{+}(n){ }^{t} \nu_{+}(m) d P=\delta_{n m} V_{+}(n) \text { and } \int_{\Omega} \nu_{-}(-n)^{t} \nu_{-}(-m) d P=\delta_{n m} V_{-}(n) \\
\mathbf{M}_{0}^{n}(\mathbf{X})=\mathbf{M}_{0}^{n}\left(\nu_{+}\right) \text {and } \mathbf{M}_{-n}^{0}(\mathbf{X})=\mathbf{M}_{-n}^{0}\left(\nu_{-}\right)
\end{gathered}
$$

We shall treat in the sequel the situation when condition (7.4) holds.

Decomposition Theorem $([35],[39])$. There exists a unique system $\left\{\gamma_{+}(n, k), \gamma_{-}(n, k)\right.$, $\left.\delta_{+}(m), \delta_{-}(m) ; 1 \leq k<n \leq N, 1 \leq m \leq N\right\}$ of $M(d ; \mathbb{R})$ such that for each $n, 1 \leq n \leq N$, the process $\mathbf{X}$ satisfies

$$
\begin{aligned}
X(n) & =-\sum_{k=1}^{n-1} \gamma_{+}(n, k) X(k)-\delta_{+}(n) X(0)+\nu_{+}(n) \\
X(-n) & =-\sum_{k=1}^{n-1} \gamma_{-}(n, k) X(-k)-\delta_{-}(n) X(0)+\nu_{-}(-n)
\end{aligned}
$$

Definition 7.1. We shall call equation (7.10) (resp. 7.11)) the forward (resp. backward) $\mathrm{KM}_{2} \mathrm{O}$-Langevin equation associated with the process $\mathbf{X}$. Further the process $\nu_{+}$(resp. $\left.\nu_{-}\right)$and the system $\left\{\gamma_{+}(n, k), \gamma_{-}(n, k), \delta_{+}(m), \delta_{-}(m) ; 1 \leq k<n \leq N, 1 \leq m \leq N\right\}$ shall be said to be the forward (resp. the backward) $K M_{2} O$-Langevin force associated with the process $\mathrm{X}$ and the $\mathrm{KM}_{2} \mathrm{O}$-Langevin data associated with the correlation matrix function $R$.

Remark 7.1. The $\mathrm{KM}_{2} \mathrm{O}$-Langevin equations were derived as the candidate for the discrete version to the $(\alpha, \beta, \gamma, \delta)$-Langevin equations which Miyoshi ([18],[19]) had derived in the continuous system. 
Now we shall observe what kinds of representations FDT stated in $\S 6$ posesess on the basis of these $\mathrm{KM}_{2} \mathrm{O}$-Langevin equations.

FDT (1) ([17],[4],[43],[45],[35],[40]). For each $n, k, 1 \leq k<n \leq N$, we have the following algorithms:

$$
\left\{\begin{array}{l}
\gamma_{+}(n, k)=\gamma_{+}(n-1, k-1)+\delta_{+}(n) \gamma_{-}(n-1, n-k-1) \\
\gamma_{-}(n, k)=\gamma_{-}(n-1, k-1)+\delta_{-}(n) \gamma_{+}(n-1, n-k-1)
\end{array}\right.
$$

$$
\left\{\begin{array}{l}
V_{+}(n)=\left(I-\delta_{+}(n) \delta_{-}(n)\right) V_{+}(n-1) \\
V_{-}(n)=\left(I-\delta_{-}(n) \delta_{+}(n)\right) V_{-}(n-1)
\end{array}\right.
$$

$$
\delta_{-}(n) V_{+}(n-1)=V_{-}(n-1)^{t} \delta_{+}(n) \quad \text { and } \quad \delta_{-}(n) V_{+}(n)=V_{-}(n){ }^{t} \delta_{+}(n)
$$

where $\gamma_{+}(n, 0)=\delta_{+}(n)$ and $\gamma_{-}(n, 0)=\delta_{-}(n)$.

Moreover we shall investigate the covariance matrix function $I(n, m)$ between the forward $\mathrm{KM}_{2} \mathrm{O}$-Langevin force $\nu_{+}$and the backward $\mathrm{KM}_{2} \mathrm{O}$-Langevin force $\nu_{-}$:

$$
I(n, m)=\int_{\Omega} \nu_{+}(n){ }^{t} \nu_{-}(-m) d P \quad(0 \leq n, m \leq N) .
$$

FDT (2) ([37]). For each $n, m, 1 \leq n \leq N, 2 \leq m \leq N$, we have

$$
\left\{\begin{aligned}
I(n, 0)= & I(0, n)=0 \\
I(n, 1)= & I(1, n)=-\delta_{+}(n+1) V_{-}(n) \\
I(n, m)= & I(n+1, m-1)+\left(\sum_{k=1}^{m-2} I(n+1, k){ }^{t} \delta_{+}(k+1)\right)^{t} \delta_{-}(m) \\
& -\delta_{+}(n+1) \sum_{k=1}^{n-1} \delta_{-}(k+1) I(k, m)
\end{aligned}\right.
$$

It follows from (7.12)-(7.14) in FDT that the system $\left\{\delta_{+}(n) ; 0 \leq n \leq N\right\}$ determines other $\mathrm{KM}_{2} \mathrm{O}$-Langevin data and it can be itself calculated in terms of the correlation function $R$ by (7.12)-(7.13) and the following algorithm: 
Algorithm ([17],[4],[43],[45],[35],[40]). For each $n, 1 \leq n \leq N$, we have

$$
\delta_{+}(n)=-\left(R(n)+\sum_{k=0}^{n-2} \gamma_{+}(n-1, k) R(k+1)\right) V_{-}(n-1)^{-1}
$$

By inverting the consideration above, assume that we are given any symmetric, positive definite matrix $V \in G L(d ; \mathbb{R})$ and any $N$ elements $\delta_{+}(n)$ of $M(d ; \mathbb{R})(1 \leq n \leq N)$.

(the first step) We construct a system $\left\{\gamma_{+}(n, k), \gamma_{-}(n, k), \delta_{-}(m), V_{+}(m), V_{-}(m) ; 1 \leq\right.$ $k<n \leq N, 1 \leq m \leq N\}$ in such a way that the relations (7.12)-(7.14) are inductively satisfied. We shall suppose in this step that all $V_{+}(m), V_{-}(m)(0 \leq m \leq N-1)$ are positive definite.

(the second step) By using Kolmogorov's extension theorem, we construct two $\mathbb{R}^{d}$ valued stochastic processes $\nu_{+}=\left(\nu_{+}(n) ; 0 \leq n \leq N\right)$ and $\nu_{-}=\left(\nu_{-}(-n) ; 0 \leq n \leq N\right)$ satisfying $\nu_{+}(0)=\nu_{-}(0)$ and relation (7.16) ([37]).

(the third step) Under the initial condition $X(0)=\nu_{+}(0)$, we solve equation (7.10) and (7.11) to obtain an $\mathbb{R}^{d}$ valued stochastic process $\mathrm{X}=(X(n) ;|n| \leq N)$.

Thus we can arrive at the following result:

Construction Theorem ([35],[40],[37]). The process $\mathbf{X}$ constructed above is a weakly stationary process with the system $\left\{\gamma_{+}(n, k), \gamma_{-}(n, k), \delta_{+}(m), \delta_{-}(m), V_{+}(m), V_{-}(m) ; 1 \leq\right.$ $k<n \leq N, 1 \leq m \leq N\}$ as its $K M_{2} O$-Langevin data.

\section{$\S 8 . \mathrm{KM}_{2} \mathrm{O}$-Langevin equations and $\mathrm{AR}(\infty)$-Langevin equations}

In this section we shall show that $\operatorname{AR}(\infty)$-Langevin equations can be derived from $\mathrm{KM}_{2} \mathrm{O}$-Langevin equations treated in $\S 7$, by a limit procedure of $N \rightarrow \infty$ ([36]).

Let $\mathrm{X}=(X(n) ; n \in \mathbb{Z})$ be an $\mathbb{R}^{d}$ valued weakly stationary process with expectation vector 0 such that its spectral matrix measure has a continuous spectral density $\Delta$ defined on $[-\pi, \pi]$. We define a one-parameter unitary group $(U(n) ; n \in \mathbb{Z})$ operating on the Hilbert space $M_{-\infty}^{\infty}(\mathrm{X})$ by

$$
U(n)\left(X_{j}(m)\right)=X_{j}(m+n)
$$


and introduce for each $N \in \mathbb{N}$ two $\mathbb{R}^{d}$ valued weakly stationary processes $\varepsilon_{+}^{N}=\left(\varepsilon_{+}^{N}(n) ; n \in\right.$ $\mathbb{Z})$ and $\varepsilon_{-}^{N}=\left(\varepsilon_{-}^{N}(n) ; n \in \mathbb{Z}\right)$ with expectation vector 0 by

$$
\varepsilon_{+}^{N}(n)=U(n-N) \nu_{+}(N) \text { and } \varepsilon_{-}^{N}(n)=U(n+N) \nu_{-}(-N)
$$

By using these processes, we find that $\mathrm{KM}_{2} \mathrm{O}$-Langevin equation (7.10) (resp. (7.11)) associated with the local and weak stationary process $(X(n) ;|n| \leq N)$ can be transformed into the following generalized $\operatorname{AR}(\infty)$-Langevin equation (8.3) (resp. (8.4)): for all $N \in$ $\mathbb{N}, n \in \mathbb{Z}$,

$$
\begin{aligned}
& X(n)=-\sum_{k=1}^{N} \gamma_{+}(N, N-k) X(n-k)+\varepsilon_{+}^{N}(n), \\
& X(n)=-\sum_{k=1}^{N} \gamma_{-}(N, N-k) X(n+k)+\varepsilon_{-}^{N}(n) .
\end{aligned}
$$

We can use the method in Grenander-Szegö ([9]) to show

Lemma 8.1. There exist two positive definite matrices $V_{+}$and $V_{-}(\in G L(d ; \mathbb{R}))$ such that

$$
\lim _{N \rightarrow \infty} V_{+}(N)=V_{+} \text {and } \lim _{N \rightarrow \infty} V_{-}(N)=V_{-}
$$

By combining (7.12)-(7.13) in FDT with (8.5), we have

Theorem 8.1. There exist two $\mathbb{R}^{d}$ valued standard white noises $\xi_{+}=\left(\xi_{+}(n) ; n \in \mathbb{Z}\right)$ and $\xi_{-}=\left(\xi_{-}(n) ; n \in \mathbb{Z}\right)$ such that

$$
\begin{aligned}
& \underset{N \rightarrow \infty}{\lim .} \varepsilon_{+}^{N}(n)=\left(V_{+}\right)^{1 / 2} \xi_{+}(n) \quad \text { and } \quad \operatorname{lij}_{N \rightarrow \infty} . \varepsilon_{-}^{N}(n)=\left(V_{-}\right)^{1 / 2} \xi_{-}(n)(n \in \mathbb{Z}), \\
& \int_{\Omega} \xi_{+}(n)^{t} \xi_{+}(m) d P=\delta_{n m} I \quad \text { and } \quad \int_{\Omega} \xi_{-}(n)^{t} \xi_{-}(m) d P=\delta_{n m} I(n, m \in \mathbb{Z}) .
\end{aligned}
$$

Applying (8.3) and (8.4) to Theorem 8.1, we obtain 
Lemma 8.2. There exists a unique system $\left\{\gamma_{+}(n), \gamma_{-}(n) ; n \in \mathbb{N}\right\}$ of $M(d ; R)$ such that for each fixed $k \in \mathbb{N}$,

$$
\lim _{N \rightarrow \infty} \gamma_{+}(N, N-k)=\gamma_{+}(k) \text { and } \lim _{N \rightarrow \infty} \gamma_{-}(N, N-k)=\gamma_{-}(k)
$$

Letting $N \rightarrow \infty$ in (8.3) and (8.4), we can make use of the above results to obtain the following forward (resp. backward) AR( $\infty$ )-Langevin equation (8.9) (resp. (8.10)).

\section{Theorem 8.2.}

$$
\begin{aligned}
& X(n)=-\underset{N \rightarrow \infty}{\lim } \sum_{k=1}^{N} \gamma_{+}(N, N-k) X(n-k)+V_{+} \xi_{+}(n) \text { a.s. } \\
& X(n)=-\underset{N \rightarrow \infty}{\lim .} \sum_{k=1}^{N} \gamma_{-}(N, N-k) X(n+k)+V_{-} \xi_{-}(n) \text { a.s. }
\end{aligned}
$$

Moreover we have the forward causal relation among $\mathbf{X}, \xi_{+}$and $\xi_{-}$.

Theorem 8.3. For each $n \in \mathbb{Z}$,

$$
\mathrm{M}_{-\infty}^{n}(\mathrm{X})=\mathrm{M}_{-\infty}^{n}\left(\xi_{+}\right) \text {and } \mathrm{M}_{n}^{\infty}(\mathrm{X})=\mathrm{M}_{n}^{\infty}\left(\xi_{-}\right)
$$

As an application of the results of this section, we shall finally lay stress on the fact that a matrix outer function $h$ of the spectral density matrix function $\Delta$ in the multidimensioanl case can be constructively obtained by the following formula.

Theorem 8.4. For a.e. $\theta \in(-\pi, \pi]$,

$$
h\left(e^{i \theta}\right)=\frac{1}{\sqrt{2 \pi}} V_{+}\left(1+\lim _{N \rightarrow \infty}\left(\sum_{k=1}^{N} \gamma_{+}(N, N-k) e^{i k \theta}\right)^{-1}\right.
$$

\section{$\S$ 9. AR-equations}

We shall observe what kinds of position AR-equations ( $\equiv$ models) which are very often used in applied fields occupy in the framework of the generalized AR-equations described in $\S 8$. 
Let $\mathrm{X}=(X(n) ; n \in \mathbb{Z})$ be an $\mathbb{R}^{d}$ valued weakly stationary process with expectation vector 0 and correlation matrix function $R$ satysfying condition (7.3). We shall fix any positive integer $N$.

Definition 9.1. We shall say that $\mathrm{X}$ is an $A R(K)$-process if and only if there exist a system $\{A(k), \sigma ; 1 \leq k \leq K\}$ of $M(d ; \mathbb{R})$ and an $\mathbb{R}^{d}$ valued standard white noise $\xi_{+}=$ $\left(\xi_{+}(n) ; n \in \mathbb{Z}\right)$ such that

$$
\begin{gathered}
X(n)=\sum_{k=1}^{K} A(k) X(n-k)+\sigma \xi_{+}(n) \quad(n \in \mathbb{Z}), \\
\sigma \in G L(d ; \mathbb{R}), \\
\int_{\Omega} \xi_{+}(n){ }^{t} \xi_{+}(m) d P=\delta_{n m} I \\
\mathbf{M}_{-\infty}^{n}(\mathbf{X})=\mathbf{M}_{-\infty}^{n}(\xi) \quad(n \in \mathbb{Z}) .
\end{gathered}
$$

We aer now in a position to show

Theorem 9.1 ([38]). The following are equivalent:

$\mathrm{X}$ is an $A R(K)$-process.

(ii) Condition (7.4) holds for all $N \in \mathbb{N}$ and $\delta_{+}(n)=0$ for all $n \geq K+1$.

(iii) Condition (7.4) holds for all $N \in \mathbb{N}$ and $M_{-\infty}^{n}\left(\varepsilon_{+}^{K}\right)=M_{-\infty}^{n}(\mathrm{X})$ for all $n \in \mathbb{Z}$.

\section{$\S 10 . \mathrm{KM}_{2} \mathrm{O}$-Langevin equations and causal relation}

The stochastic processes represent mathematical models for random phenomena varying with the change of time. It is to be noted that the meaning of the existence of causal relation among such processes has already appeared in $(3.12),(3.15),(4.10),(4.14),(7.9),(8.11)$ and (9.4). We shall in the sequel consider the situation when the stochastic processes have discrete time parameter spaces ([38]). 
Definition 10.1. (i) For given $\mathbb{R}^{d_{1}}$ (resp. $\left.\mathbb{R}^{d_{2}}\right)$ valued stochastic process $\mathrm{X}_{1}=\left(X_{1}(n) ; n \in\right.$ $\mathbb{Z})$ (resp. $\mathbf{X}_{2}=\left(X_{2}(n) ; n \in \mathbb{Z}\right)$ ), we shall say that there exists a causal relation such that $\mathbf{X}_{1}$ is a causal and $\mathbf{X}_{2}$ is a result if and only if for any $n \in \mathbb{Z}$, there exists a Borel function $F_{n}$ from $\left(\mathbb{R}^{d_{1}}\right)^{\mathbb{N}^{*}}$ to $\mathbb{R}^{d_{2}}$ such that

$$
X_{2}(n)=F_{n}\left(X_{1}(n), X_{1}(n-1), \cdots\right) \text { a.s. }
$$

(ii) In particular we shall say that the causal relation between $\mathbf{X}_{1}$ and $\mathbf{X}_{2}$ is linear (resp. non-linear of $p$ th order) according to the case where the functions $F_{n}$ can be taken as polinomials of the first degree (resp. $p(\geq 2)$ th degree). In this case we shall write $\mathrm{X}_{1} \stackrel{\text { linear }}{\longrightarrow} \mathrm{X}_{2}, \mathrm{X}_{1} \stackrel{\text { non-linear of } p \text { th order }}{\longrightarrow} \mathrm{X}_{2}$, respectively.

Putting $d_{1}=d$ and $d_{2}=1$, we shall assume that the $\mathbb{R}^{d+1}$ valued stochastic process $\mathrm{X}=\left({ }^{t}\left({ }^{t} X_{1}(n), X_{2}(n)\right) ; n \in \mathbb{Z}\right)$ formed by an arrangement of $\mathrm{X}_{1}$ and $\mathrm{X}_{2}$ is weakly stationary with expectation vector 0 . We define three kinds of correlation matrix functions $R_{1}, R_{2}$ and $R_{3}$ by

$$
\left\{\begin{array}{l}
R_{1}(n)=\int_{\Omega} X_{1}(n)^{t} X_{1}(0) d P \in M(d, d ; \mathbb{R}) \\
R_{2}(n)=\int_{\Omega} X_{2}(n)^{t} X_{1}(0) d P \in M(1, d ; \mathbb{R}) \\
R_{3}(n)=\int_{\Omega} X_{2}(n) X_{2}(0) d P \in M(1,1 ; \mathbb{R}) .
\end{array}\right.
$$

Then we have a fundamental principle.

Theorem 10.1. The following (i) and (ii) is equivalent:

$$
\mathrm{X}_{1} \stackrel{\text { linear }}{\longrightarrow} \mathrm{X}_{2} \text {. }
$$

$$
C_{n}\left(\mathbf{X}_{2} \mid \mathbf{X}_{1}\right) \text { converges increasingly to } R_{3}(0) \text { as } n \rightarrow \infty \text {. }
$$

Here the function $C_{n}\left(\mathbf{X}_{2} \mid \mathbf{X}_{1}\right)$ stands for the causality function from $\mathbf{X}_{1}$ to $\mathbf{X}_{2}$ defined by

$$
C_{n}\left(\mathbf{X}_{2} \mid \mathbf{X}_{1}\right) \equiv\left(\int_{\Omega}\left(P_{\mathbf{M}_{0}^{n}\left(\mathbf{X}_{1}\right)} X_{2}(n)\right)^{2} d P\right)^{1 / 2}
$$

For the purpose of calculating this causality function, we shall make use of the $\mathrm{KM}_{2} \mathrm{O}$ Langevin random force $\nu_{+}$and the $\mathrm{KM}_{2} \mathrm{O}$-Langevin data $\left\{\gamma_{+}(n, k), V_{+}(k) ; 0 \leq k<n<\right.$ 
$\infty$ \} associated wit the weakly stationary process $\mathbf{X}_{1}$. It follows from a local causal relation (7.9) between $\mathrm{X}_{1}$ and $\nu_{+}$that there exists a system $\{C(n, k) ; 0 \leq k \leq n<\infty\}$ of $M(1, d ; \mathbb{R})$ such that

$$
P_{\mathrm{M}_{0}^{n}\left(\mathrm{X}_{1}\right)} X_{2}(n)=\sum_{k=0}^{n} C(n, k) \nu_{+}(k) \quad\left(n \in \mathbb{N}^{*}\right)
$$

Thus we can arrive at the following formula.

Theorem 10.2. For any $n, k, 0 \leq k \leq n$,

$$
\begin{gathered}
C_{n}\left(\mathbf{X}_{2} \mid \mathbf{X}_{1}\right)=\left\{\sum_{k=0}^{n} C(n, k) V_{+}(k){ }^{t} C(n, k)\right\}^{1 / 2}, \\
C(n, k)= \begin{cases}R_{2}(n) R_{1}(0)^{-1} & (k=0) \\
\left\{R_{2}(n-k)+\sum_{l=0}^{k-1} R_{2}(n-l){ }^{t} \gamma_{+}(k, l)\right\} V_{+}(k)^{-1} & (k \geq 1) .\end{cases}
\end{gathered}
$$

Remark 10.1. In the case where $d=1$, we can treat the problem of non-linear causal relation of $p$ th order from $\mathbf{X}_{1}$ to $\mathbf{X}_{2}$ by considerating the one of linear causal relation from $\mathrm{X}_{3}=\left({ }^{t}\left(X_{1}(n), X_{1}(n)^{2}, \cdots, X_{1}(n)^{p}\right) ; n \in \mathbb{Z}\right)$ to $\mathrm{X}_{2}$.

\section{$\S 11$. Stationary analysis and causal analysis}

When we are given two kinds of data, we shall at first consider the time series formed by them and apply our theory to them to propose two tests: Test(S) and Test(CS); Test(S) is a stationary test which checks the local and weak stationarity of them and Test(CS) is a causal test which discriminates the existence and direction between given two kinds of data. Furthermore we shall discuss their effectiveness ([38],[39]).

[11.1] (Stationary analysis) Let us given an $\mathbb{R}^{d}$ valued data $\mathcal{Z}=(\mathcal{Z}(n) ; 0 \leq n \leq$ $N)$.

(the first step) We define a sample mean vector $\mu^{\mathcal{E}}$ and a sample correlation matrix function $R^{\mathcal{Z}}=\left(R_{j k}^{\mathcal{Z}}(*)\right)_{1 \leq j, k \leq d}$ as follows:

$$
\mu^{\mathcal{E}} \equiv \frac{1}{N+1} \sum_{n=0}^{N} \mathcal{Z}(n)
$$




$$
\left\{\begin{array}{l}
R_{j k}^{\mathcal{Z}}(n) \equiv \frac{1}{N+1} \sum_{m=0}^{N-n}\left(\mathcal{Z}_{j}(n+m)-\mu_{j}^{\mathcal{Z}}\right)\left(\mathcal{Z}_{k}(m)-\mu_{k}^{Z}\right) \\
R_{j k}^{\mathcal{Z}}(-n) \equiv R_{k j}^{\mathcal{Z}}(n),
\end{array}\right.
$$

where $\mu^{\mathcal{Z}}={ }^{t}\left(\mu_{1}^{\mathcal{Z}}, \cdots, \mu_{d}^{\mathcal{Z}}\right), \mathcal{Z}={ }^{t}\left(\mathcal{Z}_{1}(n), \cdots, \mathcal{Z}_{d}(n)\right) \quad(0 \leq n \leq N)$.

(the second step) The standardized data $\mathcal{X}={ }^{t}\left(\mathcal{X}_{1}(n), \cdots, \mathcal{X}_{d}(n)\right)$ of $\mathcal{Z}$ is defined by

$$
\mathcal{X}_{j}(n) \equiv\left(R_{j j}^{\mathcal{Z}}(0)\right)^{-1 / 2}\left(\mathcal{Z}_{j}(n)-\mu_{j}^{\mathcal{E}}\right) \quad(1 \leq j \leq d, 0 \leq n \leq N) .
$$

We denote by $R^{\mathcal{X}}(n)(0 \leq n \leq N)$ its sample correlation matrix function. It follows from an experiance rule in data analysis ([1]) that the maximal number $M$ such that the sample correlation function $R^{\mathcal{X}}(n)(0 \leq n \leq M)$ becomes our reliable data is given by

$$
M \equiv[3 \sqrt{N+1} / d]-1
$$

(the third step) The function $R^{\mathcal{X}}(n)(-M \leq n \leq M)$ obtained above can be extended as a non-negative definite function, e.g. by letting $R^{X}(n)=0$ for $|n| \geq M+1$. By using the same way as in the construction theorem (the first step) in $\S 7$, therefore, we can replace the function $R$ in the algorithm (7.17) by the sample correlation function $R^{\mathcal{X}}$ to construct a sample $\mathrm{KM}_{2} \mathrm{O}$-Langevin data $\left\{\gamma_{+}(n, k), \gamma_{-}(n, k), \delta_{+}(m), \delta_{-}(m), V_{+}(m), V_{-}(m)\right.$; $1 \leq k<n \leq M, 1 \leq m \leq M\}$ associated with $R^{x}$. In this procedure we assume that all the matrices $V_{+}(m), V_{-}(m)(0 \leq m \leq M-1)$ are positive definite.

(the fourth step) We shall replace $X(n)$ by $\mathcal{X}(n)$ in the forward $\mathrm{KM}_{2} \mathrm{O}$-Langevin equation (7.10) to construct a sample $\mathrm{KM}_{2} \mathrm{O}$-Langevin random force $\nu_{+}=\left(\nu_{+}(n) ; 0 \leq\right.$ $n \leq M)$ by

$$
\left\{\begin{array}{l}
\nu_{+}(0) \equiv \mathcal{X}(0) \\
\nu_{+}(n) \equiv \mathcal{X}(n)+\sum_{k=0}^{n-1} \gamma_{+}(n, k) \mathcal{X}(k) \quad(1 \leq n \leq M) .
\end{array}\right.
$$

By taking $M+1$ elements $W_{+}(n)$ of $G L(d ; \mathbb{R})$ such that

$$
V_{+}(n)=W_{+}(n){ }^{t} W_{+}(n) \quad(0 \leq n \leq M),
$$

we shall proceed to a standardization of $\xi_{+}=\left(\xi_{+}(n) ; 0 \leq n \leq M\right)$ of $\nu_{+}$:

$$
\xi_{+}(n) \equiv W_{+}(n)^{-1} \nu_{+}(n)={ }^{t}\left(\xi_{+1}(n), \cdots, \xi_{+d}(n)\right) .
$$


Moreover we shall form $d(M+1)$ components of $d$-dimensional data $\xi_{+}$into line and construct a one-dimensional data $\xi=(\xi(n) ; 0 \leq n \leq d(M+1)-1)$ as follows:

$$
\xi(n) \equiv \xi_{+j}(m), \quad n=d m+j-1(1 \leq j \leq d, 0 \leq m \leq M) .
$$

(the fundamental principle) By taking account of the decomposition theorem, FDT and the construction theorem in $\S 7$, we find that the following (11.10) and (11.11) are equivalent each other:

$$
\left\{\begin{array}{l}
\mathcal{X} \text { is a realization of a local and weak stationary process with } R^{\mathcal{X}} \\
\text { as its correlation matrix function. }
\end{array}\right.
$$

$$
\xi \text { is a realization of a standard white noise. }
$$

(the fifth step) Since only a set of original data $\mathcal{X}$ is insufficient for checking condition (11.10), we shall take $N-M+1$ kinds of data $\mathcal{X}_{i}(0 \leq i \leq N-M)$, which can be regarded as the same kind as the data $\mathcal{X}$, from the data $\mathcal{X}$ with lengh $N+1$ as follows:

$$
\mathcal{X}_{i} \equiv(\mathcal{X}(i+n) ; 0 \leq n \leq M)
$$

Similarly as $\xi$ in (11.9), we shall construct for any $i, 0 \leq i \leq N-N$, a one-dimensional data $\xi_{i}=\left(\xi_{i}(n) ; 0 \leq n \leq d(M+1)-1\right)$ by replacing $X(n)$ with $\mathcal{X}_{i}(n)$. It is our aim to investigate whether these data satisfy the fundamental principle (11.11). For that purpose, by making use of the central limit theorem and the law of large numbers, we shall propose three kinds of tests $(M)_{i},(V)_{i}$ and $(O)_{i}:(M)_{i}$ is a test for mean $0,(V)_{i}$ for variance 1 and $(O)_{i}$ for orthogonality; for each $n, m, 1 \leq n \leq M, 0 \leq m \leq M-n$,

$$
\begin{gathered}
\sqrt{d(M+1)}\left|\mu^{\xi_{i}}\right|<1.96 \\
\left|\left(v^{\xi_{i}}-1\right)^{\sim}\right|<2.2414 \\
d(M+1)\left(\sum_{j=1}^{2}\left(L_{n, m}^{(j)}\right)^{-1 / 2}\left|R^{\xi_{i}}(n, m)\right|\right)<1.96
\end{gathered}
$$$$
(O) i
$$ 
where

$$
\begin{gathered}
\mu^{\xi_{i}} \equiv \frac{1}{d(M+1)} \sum_{k=0}^{d(M+1)-1} \xi_{i}(k) \\
\left(v^{\xi_{i}}-1\right)^{\sim} \equiv\left\{\sum_{k=0}^{d(M+1)-1}\left(\xi_{i}(k)^{2}-1\right)\right\}\left\{\sum_{k=0}^{d(M+1)-1}\left(\xi_{i}(k)^{2}-1\right)^{2}\right\}^{-1 / 2} \\
R^{\xi_{i}}(n, m) \equiv \frac{1}{d(M+1)} \sum_{k=m}^{d(M+1)-1-n} \xi_{i}(k) \xi_{i}(n+k)
\end{gathered}
$$

Moreover the numbers $L_{n, m}^{(j)}$ are determined as follows: we denote by $r$ the residue by dividing $d(M+1)$ by $2 n$ and set $[m / n]=s$;

(i) if $0 \leq r \leq n$,

$$
\left\{\begin{array}{l}
L_{n, m}^{(1)}= \begin{cases}n(q+(s / 2))-m \\
n(q-(s+1) / 2) & (\text { s even })\end{cases} \\
L_{n, m}^{(2)}= \begin{cases}n(q-1-(s / 2))+r & (\text { s odd }) \\
n(q-1+(s+1) / 2)+r-m & (\text { s ode })\end{cases}
\end{array}\right.
$$

(ii) if $n+1 \leq r \leq 2 n-1$,

$$
\left\{\begin{array}{l}
L_{n, m}^{(1)}= \begin{cases}n(q-1+(s / 2))+r-m & (s \text { even }) \\
n(q-1-(s+1) / 2)+r & (s \text { odd })\end{cases} \\
L_{n, m}^{(2)}= \begin{cases}n(q-(s / 2)) & \text { (s even) } \\
n(q+(s+1) / 2)-m & \text { (s odd }) .\end{cases}
\end{array}\right.
$$

It is to be noted that these numbers $L_{n, m}^{(j)}$ satisfy the following:

$$
d(M+1)-n-m=L_{n, m}^{(1)}+L_{n, m}^{(2)}
$$

(the sixth step) We assert that the given data $\mathcal{Z}$ is a realization of a local and weak stationary process when the following Test(S) is passed ([39]):

(S) $\left\{\begin{array}{l}\text { The rate of numbers } i(0 \leq i \leq N-M) \text { such that Test }\left((M)_{i}\right) \text {, Test }\left((V)_{i}\right) \text { and } \\ \text { Test }\left((O)_{i}\right) \text { pass through is more than } 80 \%, 70 \% \text { and } 80 \% \text {, respectively. }\end{array}\right.$ 
This is an experiance rule which can be derived from a lot of experiments by using various kinds of data whose law and structure are well known. We will regret not to be able to manifest it mathematically. It can be obtained as a resut of pushing an applied mathematical study forward, with our definite object to check whether the data has a local and weak stationarity, according to our guidance principle FDP. It seems that there can be found certain importance, difficulty and interest of applied mathematics.

[11.2] (Causal analysis) Finally we shall apply the results in $\S 10$ to causal analysis. For a data $\mathcal{Z}_{1}=\left(\mathcal{Z}_{1}(n) ; 0 \leq n \leq N\right)$ formed by $N+1$ numbers of $\mathbb{R}^{d}$ and a data $\mathcal{Z}_{2}=\left(\mathcal{Z}_{2}(n) ; 0 \leq n \leq N\right)$ formed by $N+1$ numbers of $\mathbb{R}$, we shall consider the question of linear causality: $\mathcal{Z}_{1} \stackrel{\text { linear }}{\longrightarrow} \mathcal{Z}_{2}$.

(the seventh step) We shall consider the situation when the $\mathbb{R}^{d+1}$ valued data $\mathcal{Z}=$ $\left({ }^{t}\left({ }^{t} \mathcal{Z}_{1}(n), \mathcal{Z}_{2}(n)\right) ; 0 \leq n \leq N\right)$ passes Test(S). We denote by $\mathcal{X}=\left({ }^{t}\left({ }^{t} \mathcal{X}_{1}(n), \mathcal{X}_{2}(n)\right) ; 0 \leq\right.$ $n \leq N)$ the standardized data of $\mathcal{Z}$. It is to be noted that the number $M$ in (11.4) is $[3 \sqrt{N+1} /(d+1)]-1$ in this case. Furthermore we denote by $R^{\mathcal{X}_{1}}, R^{\mathcal{X}_{2} \mathcal{X}_{1}}$ and $R^{\mathcal{X}_{2}}$ three functions $R_{1}, R_{2}$ and $R_{3}$ in (10.2), respectively. Let the system $\left\{\gamma_{+}(n, k), \gamma_{-}(n, k), \delta_{+}(m)\right.$, $\left.\delta_{-}(m), V_{+}(m), V_{-}(m) ; 1 \leq k<n \leq M, 1 \leq m \leq M\right\}$ be the sample $\mathrm{KM}_{2} \mathrm{O}$-Langevin data associated with the data $R^{\mathcal{X}_{1}}$.

(the eighth step) By replacing $R_{1}$ and $R_{2}$ in (10.5) and (10.6) with $R^{X_{1}}$ and $R^{x_{2} x_{1}}$, respectively, we shall draw up a graph of the function $C_{n}\left(\mathcal{X}_{2} \mid \mathcal{X}_{1}\right)$. Noting $R^{\mathcal{X}_{2}}(0)=1$, we shall assert ([38]) that

$$
\left\{\begin{array}{l}
\text { there exists a linear causality }: \mathcal{Z}_{1} \stackrel{\text { linear }}{\longrightarrow} \mathcal{Z}_{2} \text { between both the data } \mathcal{Z}_{1} \text { and } \\
\text { the data } \mathcal{Z}_{2} \text { when the sample causality function } C_{n}\left(\mathcal{X}_{2} \mid \mathcal{X}_{1}\right)(0 \leq n \leq M) \\
\text { from } \mathcal{X}_{1} \text { to } \mathcal{X}_{2} \text { has a tendency to increase to } 1 .
\end{array}\right.
$$

Remark 11.1. By using the same idea as in Remark 10.1, we can discuss the question of the existence of non-linear causal relation of $p$ th order between the one-dimensional data $\mathcal{Z}_{1}$ and $\mathcal{Z}_{2}$.

\section{$\S 12$. Causal relation among meteorological data}

[12.1] We shall construct a dynamics by using some data whose rule are well known and investigate certain causal relation among them to observe the aspect of sample causality functions. 
For a one-dimensional data $\mathcal{Z}=(\mathcal{Z}(n) ; 0 \leq n \leq 104)$, we shift it into

$$
\mathcal{Y}(n)=\mathcal{Z}(n+5) \quad(-5 \leq n \leq 99)
$$

and define a dynamics made from five kinds of data $\mathcal{Z}_{j}=\left(\mathcal{Z}_{j}(n) ; 0 \leq n \leq 99\right)(1 \leq j \leq 5)$ :

$$
\left\{\begin{array}{l}
\mathcal{Z}_{1}(n)=\mathcal{Y}(n) ; \mathcal{Z}_{2}(n)=\mathcal{Y}(n)^{2} ; \mathcal{Z}_{3}(n)=\mathcal{Y}(n)^{3} \\
\mathcal{Z}_{4}(n)=\mathcal{Y}(n-3)-2 \mathcal{Y}(n-5) ; \mathcal{Z}_{5}(n)=\mathcal{Y}(n-3)-2 \mathcal{Y}(n-5)^{2}
\end{array}\right.
$$

As the data $\mathcal{Z}$, we adopt an $\operatorname{AR}(2)$-data which can be obtained in the construction theorem in $\$ 7$ by choosing

$$
(\mathrm{v}, \delta(1), \delta(2))=(1,0.6,-0.3) \text { and } \delta(n)=0(n \geq 3)
$$

By Theorem 9.1, this can be also mentioned as follows. We at first take a standardized uniform number $\xi=(\xi(n) ; 0 \leq n \leq 104)$ and then calculate the following:

$$
\mathcal{Z}(n)= \begin{cases}-\sum_{k=0}^{n-1} \gamma(n, k) \mathcal{Z}(k)+\sqrt{V(n)} \xi(n) & (0 \leq n \leq 2) \\ -\sum_{k=1}^{2} \gamma(2,2-k) \mathcal{Z}(n-k)+\sqrt{V(2)} \xi(n) & (3 \leq n \leq 104)\end{cases}
$$

As the data $\xi$, we make use of 100 standardized uniform numbers with seed number from 2 to 541 and construct 10 kinds of two-dimensional data $\left({ }^{t}\left(\mathcal{Z}_{j}(n), \mathcal{Z}_{k}(n)\right) ; 0 \leq n \leq\right.$ 99) $(1 \leq j<k \leq 5)$. We show in Table 12.1 the results of Test(S) for them:

\begin{tabular}{|c|c|c|c|c|}
\hline & $\mathcal{Z}_{2}$ & $\mathcal{Z}_{3}$ & $\mathcal{Z}_{4}$ & $\mathcal{Z}_{5}$ \\
\hline $\mathcal{Z}_{2}$ & 0.93 & 0.74 & 0.70 & 0.97 \\
\hline $\mathcal{Z}_{3}$ & & $0.23^{*}$ & 0.99 & $0.58^{*}$ \\
\hline $\mathcal{Z}_{4}$ & & & 0.93 & $0.54^{*}$ \\
\hline $\mathcal{Z}_{5}$ & & & & 0.97 \\
\hline
\end{tabular}

\begin{tabular}{|l|c|c|}
\hline & Arct $\mathcal{Z}_{3}$ & Arct $\mathcal{Z}_{5}$ \\
\hline Arct $\mathcal{Z}_{2}$ & 0.83 & 0.97 \\
\hline Arct $\mathcal{Z}_{3}$ & & 0.98 \\
\hline
\end{tabular}

Table 12.1 Test(S)

Table 12.2 Test(S)

We can not find any desirable result in Test(S) for two-dimensional data formed by taking any pairings from $\mathcal{Z}_{2}, \mathcal{Z}_{3}$ and $\mathcal{Z}_{5}$. The reason for this seems to lie in the multiplication effect that they are made from taking the square and the tube of the data $\mathcal{Y}$. In order to remove it, we apply arctangent transformation to each of them and define new data $\operatorname{Arct} \mathcal{Z}_{2}, \operatorname{Arct} \mathcal{Z}_{3}$ and $\operatorname{Arct} \mathcal{Z}_{5}$. The results of Test(S) for these are shown in Table 12.2 with a good result.

Since our data were shown to have a local and weak stationarity, we can proceed to Test(CS). The results are illustrated in Figure 12.1-Figure 12.3. These teach us that the 
test of linear causality is useless for the data which are combined with certain non-linear relations and any test of no-linear causality is also ineffective if the degree $p$ of non-linearlity is different.

Figure 12.1 Test(CS)

Figure 12.2 Test(CS)

Figure 12.3 Test(CS)

[12.2] Let $\mathcal{Z}_{6}=\left(\mathcal{Z}_{6}(n) ; 0 \leq n \leq 113\right)$ and $\mathcal{Z}_{7}=\left(\mathcal{Z}_{7}(n) ; 0 \leq n \leq 113\right)$ be Wolfer's sunspot numbers and the trapped numbers of Canadian Lynx in Mackenzie River from the period 1821 to 1934 , respectively $([12],[46],[7])$. We show in Table 12.3 and Figure 12.4 the results of Test(S) and Test(CS) for two-dimensional data made from $\mathcal{Z}_{6}$ and $\mathcal{Z}_{7}$, respectively.

\begin{tabular}{|c|c|c|c|c|}
\hline & $(\mathrm{M})$ & $(\mathrm{V})$ & $(\mathrm{O})$ & $(\mathrm{S})$ \\
\hline${ }^{t}\left(\mathcal{Z}_{6}, \mathcal{Z}_{7}\right)$ & 0.980 & 0.949 & 0.919 & $\mathrm{~S}$ \\
\hline
\end{tabular}

Table 12.4 Test(CS)

\section{Figure 12.4}

Figure 12.4 Test(CS)

We can obtain similar results also when we apply both logarithmic and arctangent transformations to the original data $\mathcal{Z}_{6}$ and $\mathcal{Z}_{7}$. Though only these transformations are insufficient for certain objective assertion, we can not say from the comparison with Figure 12.1-Figure 12.3 that there exists certain marked causal relation between Wolfer's sunspot numbers and the trapped numbers of Canadian Lynx in MacKenzie River.

[12.3] Let $\mathcal{Z}_{8}=\left(\mathcal{Z}_{8}(n) ; 0 \leq n \leq 99\right), \mathcal{Z}_{9}=\left(\mathcal{Z}_{9}(n) ; 0 \leq n \leq 99\right)$ and $\mathcal{Z}_{10}=$ $\left(\mathcal{Z}_{10}(n) ; 0 \leq n \leq 99\right)$ be Wolfer's sunspot numbers, the rainfall averages in a year in Sapporo and the temperature averages in a year in Sapporo from the period 1889 to 1988. We show the results of Test(S) for three kinds of two-dimensional data formed by these data.

\begin{tabular}{|c|c|c|c|c|}
\hline & $(\mathrm{M})$ & $(\mathrm{V})$ & $(\mathrm{O})$ & $(\mathrm{S})$ \\
\hline${ }^{t}\left(\mathcal{Z}_{8}, \mathcal{Z}_{9}\right)$ & 0.977 & 0.860 & 1.000 & $\mathrm{~S}$ \\
\hline${ }^{t}\left(\mathcal{Z}_{8}, \mathcal{Z}_{10}\right)$ & 0.988 & $0.674^{*}$ & 0.988 & NS \\
\hline${ }^{t}\left(\mathcal{Z}_{9}, \mathcal{Z}_{10}\right)$ & 1.000 & 0.872 & 0.988 & $\mathrm{~S}$ \\
\hline $\mathcal{Z}_{5}$ & & & & 0.97 \\
\hline
\end{tabular}

Table 12.4 Test(S) 


\begin{tabular}{|c|c|c|c|c|}
\hline & $(\mathrm{M})$ & $(\mathrm{V})$ & $(\mathrm{O})$ & $(\mathrm{S})$ \\
\hline${ }^{t}\left(\right.$ Arct $\mathcal{Z}_{8}$, Arct $\left.\mathcal{Z}_{9}\right)$ & 1.000 & 1.000 & 1.000 & $\mathrm{~S}$ \\
\hline${ }^{t}\left(\right.$ Arct $\mathcal{Z}_{8}$, Arct $\left.\mathcal{Z}_{10}\right)$ & 1.000 & 0.953 & 0.965 & $\mathrm{~S}$ \\
\hline${ }^{t}\left(\right.$ Arct $\mathcal{Z}_{9}$, Arct $\left.\mathcal{Z}_{10}\right)$ & 0.988 & 0.907 & 0.977 & $\mathrm{~S}$ \\
\hline
\end{tabular}

Table 12.5 Test(S)

The test of local and weak stationarity is rejected for the data $\mathcal{Z}_{8}$ and $\mathcal{Z}_{10}$. If we apply arctangent transformation to them, however, we find that new data pass Test(S) whose results are shown in Table 12.5 .

Therefore, we can proceed to Test(CS) for the data transformed by arctangent transform. The results are illustrated in Figure 12.5-Figure 12.7.

Figure 12.5 Test(CS)

Figure 12.6 Test(CS)

Figure 12.7 Test(CS)

It is likely that Wolfer's sunspot numbers causes the temperature in a year in Sapporo and the rainfall in a year in Sapporo causes the temperature in a year in Sapporo. However, we have to abstain from concluding from Figure 12.5 whether there exists certain causal relation between Wolfer's sunspot numbers and the rainfall in a year in Sapporo.

Finally we would like to lay down our pen by stating some tasks remained to be solved. It is very important for us to carry Test(CS) forward to give a systematic treatment for the non-linear structure in random phenomena, because it relates with the settlement of non-linear prediction problems by obtaining certain algorithms which are usefull in applied fields. We are now under investigation to perform such a study. Moreover we intend to apply our theory to some important economic time series and compare the results on the basis of our theory with those based upon the causal analysis of economic time series due to Granger ([8]). Furthermore we think that the problem to quantify our decision standard for Test(CS) has to be overcome by all means in order that we can carry forward our project for data analysis of time series under our philosophy FDP. 
Notes in translation. We have recently solved the problem of non-linear prediction for one-dimensional strictly stationary processes in the following two papers: Applications of the theory of $\mathrm{KM}_{2} \mathrm{O}$-Langevin equations to the linear prediction problem for the multidimensional weakly stationary time series, to be submitted in J. Math. Soc. Japan and Applications of the theory of $\mathrm{KM}_{2} \mathrm{O}$-Langevin equations to the non-linear prediction problem for the one-dimensional strictly stationary time series, to be submitted in Acta. Math..

\section{References}

[1]. H. Akaike and T. Nakagawa, "Statistical analysis and control of dynamics," Science, 1972.

[2]. Alder, B.J. and T.E. Wainwright, Velocity autocorrelations for hard spheres, Phys. Rev. Lett. 18 (1967), 988-990.

[3]. Boussinesq, J., Sur la résistance qu'oppose un liquide indéfini en repos, sans pesanteur, au mouvement varié d'une sphère solide, C.R. Acad. Sci. Paris 100 (1885), 935-937.

[4]. Durbin, J., The fitting of time series models, Rev. Int. Stat. 28 (1960), 233-244.

[5]. Dym, H. and H.P. McKean, Jr., "Gaussian Processes, Function Theory and the Inverse Spectral Problem," Academic Press, 1976.

[6]. Einstein, A., Über die von der molekularkinetischen Theorie der Wärme geforderte Bewegung von in ruhenden Flüssigkeiten suspendierten Teilchen, Drudes Ann. 17 (1905), 549-560.

[7]. Elton, C. and M.Nicholson, The ten-year cycle in numbers of the Lynx in Canada, J. Anim. Ecol. 11 (1942), 215-244.

[8]. Granger, C.W.J., Investigating causal relations by econometric models and crossspectral methods, Econometrica 37 (1969), 424-438.

[9]. Grenander, U. and G. Szegö, "Toeplitz forms and their applications," University of California Press, Berkeley and Los Angeles, 1958.

[10]. Inoue, A., The Alder-Wainwright effect for stationary processes with reflection positivity (I), J. Math. Soc. Japan 43 (1991), 515-526.

[11]. The Alder-Wainwright effect for stationary processes with reflection positivity (II), Osaka J. Math. 28 (1991), 537-561. 
[12]. Jevons, W.S., Commercial crises and sun-spots, Nature 15 (1878), 33-37.

[13]. Kubo, R., Statistical mechanical theory of irreversible processes $I$, general theory and sipmple applications to magnetic and conduction problem, J. Phys. Soc. Japan 12 (1957), 570-586.

[14]. The fluctuation-dissipation theorem, Rep. Progr. Phys. 29 (1966), 255-284.

$[15]$. Irreversible processes and Stochastic processes, The theory of stochastic processes and Statistical mechanics of open system (R.I.M.S., Kôkyuroku) 367 (1979), $50-93$.

[16]. Langevin, P., Sur la théorie du mouvement brownien, C.R.Acad.Sci.Paris 146 (1908), $530-533$.

[17]. Levinson, N., The Wiener RMS error criterion in filter design and prediction, J. Math. Phys. 25 (1947), 261-278.

[18]. Miyoshi, T., On $(l, m)$-string and $(\alpha, \beta, \gamma, \delta)$-Langevin equation associated with a stationary Gaussian process, J. Fac. Sci. Univ. Tokyo, SectIA. 30 (1983), 139-190. [19]. On an $\mathbb{R}^{d}$-valued stationary Gaussian process associated with $(k, l, m)$ string and $(\alpha, \beta, \gamma, \delta)$-Langevin equation, J. Fac. Sci. Univ. Tokyo, SectIA. 31 (1984), 154-194.

[20]. Nakano, Y. and Y. Okabe, On a multi-dimensional $[\alpha, \beta, \gamma]$-Langevin equation, Proc. Japan Acad. 59 (1983), 171-173.

[21]. Okabe, Y., On a stationary Gaussian process with T-positivity and its associated Langevin equation and S-matrix, J. Fac. Sci. Univ. Tokyo, SectIA. 26 (1979), 115-165. [22]. — On a stochastic differential equation for a stationary Gaussian process for a stationary Gaussian process with T-positivity and the fluctuation-dissipation theorem, J. Fac. Sci. Univ. Tokyo, SectIA. 28 (1981), 169-213.

[23]. —_ On a stochastic differential equation for a stationary Gaussian process for a stationary Gaussian process with finite multiple Markovian property and the fluctuation-dissipation theorem, J. Fac. Sci. Univ. Tokyo, SectIA. 28 (1982), 793-804. $[24]$. On a Langevin equation, Sügaku 33 (1981), 306-324.

[25]. - On a wave equation associated with prediction errors for a stationary Gaussian process, Lecture Notes in Control and Information Sciences 49 (1983), 215-226. 
[26]. A generalized fluctuation-dissipation theorem for the one-dimensional diffusion process, Commun. Math. Phys. 98 (1985), 449-468.

[27]. On KMO-Langevin equations for stationary Gaussian processes with $T$. positivity, J. Fac. Sci. Univ. Tokyo, SectIA. 33 (1986), 1-56.

[28]. On the theory of Brownian motion with the Alder-Wainwright effect, J. Stat. Phys. 45 (1986), 953-981.

[29]. KMO-Langevin equation and fluctuation-dissipation theorem (I), Hokkaido Math. J. 15 (1986), 163-216.

[30]. KMO-Langevin equation and fluctuation-dissipation theorem(II), Hokkaido Math. J. 15 (1986), 317-355. , On long time tails of correlation functions for KMO-Langevin equations, Proceedings of the Fourth Japan-USSR Symposium on Probability and Mathematical Statistics, Kyoto, Lecture Notes in Mathematics, Springer, Tokyo 1299 (1986), 391-397.

[32]. On the theory of discrete KMO-Langevin equations with reflection positivity (I), Hokkaido Math. J. 16 (1987), 315-341. [33]. , On the theory of discrete KMO-Langevin equations with reflection positivity (II), Hokkaido Math. J. 17 (1988), 1-44.

[34]. - On the theory of discrete KMO-Langevin equations with reflection positivity (III), Hokkaido Math. J. 18 (1989), 149-174.

[35]. — On stochastic difference equations for the multi-dimensional weakly stationary time series, in "Prospect of Algebraic Analysis(ed. by M. Kashiwara and T. Kawai)," Academic press, Tokyo, 1988, pp. 601-645.

[36]. — The random forces associated with discrete-time weakly stationary processes, Proceedings of Preseminar for International Conference on Gaussian Random fields,Nagoya,1991,126-134.

[37]. — $\longrightarrow \mathrm{K}_{2} \mathrm{O}$-Langevin equation and the fluctuation-dissipation theorem (in preparation).

[38]. Okabe,Y. and A. Inoue, On the exponential decay of the correlation functions for KMO-Langevin equations, to appear in Japan. J. Math.. 
[39]. The theory of $\mathrm{KM}_{2} \mathrm{O}$-Langevin equations and its applications to data analysis (II): Causal analysis, to be submitted in Nagoya Math. J..

[40]. Okabe, Y. and Y. Nakano, The theory of $\mathrm{KM}_{2} \mathrm{O}$-Langevin equations and its applications to data analysis (I): Stationay analysis, Hokkaido Math. J. 20 (1991), 45-90.

[41]. Oobayashi, K., Kohno, T. and H. Utiyama, Photon correlation spectroscopy of the non-Markovian Brownian motion of spherical particles, Phys. Rev. A 27 (1983), 2532-2641.

[42]. Stokes, G.G., On the effect of the internal friction of fluids on the motion of pendulums, Trans. Cambridge Philos. Soc. 9 (1856). pt.2, 8-106

[43]. Whittle, $\mathrm{P}$, On the fitting of multivariate autoregressions, and the approximate canonical factorization of a spectral density matrix, Biomerika 50 (1963), 129-134.

[44]. Widom, A., Velocity fluctuations of a hard-core Brownian motion, Phys. Rev. A 3 (1971), 1394-1396.

[45]. Wiggins, R.A. and E.A. Robinson, Recursive solution to the multichannel fitting problem, J. Geophys. Res. 70 (1965), 1885-1891.

[46]. Yule, G.U., On a method of investigating periodicities in disturbed series, with Wolfer's sunspot numbers, Phil. Trans. Roy. Soc. London, Ser. A 226 (1927), 268-298. 


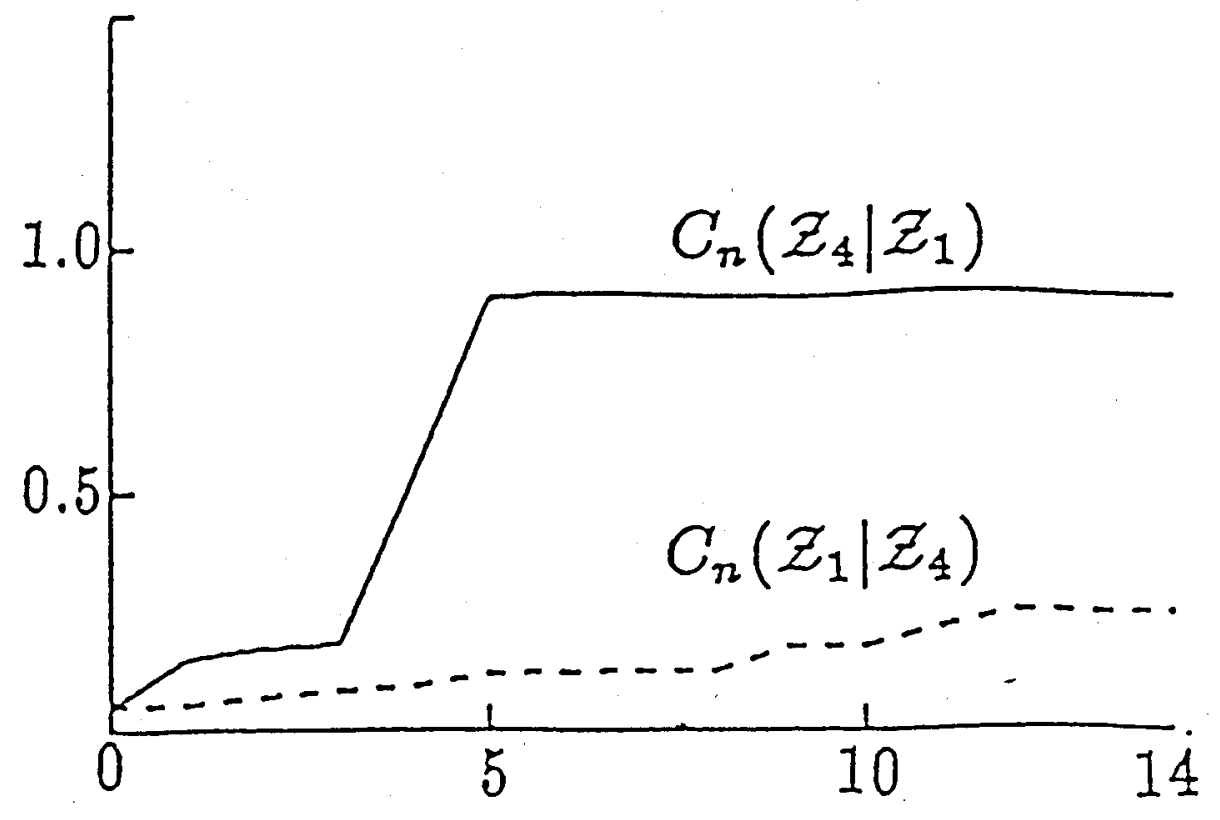

Figure 12.1 Test(CS) 


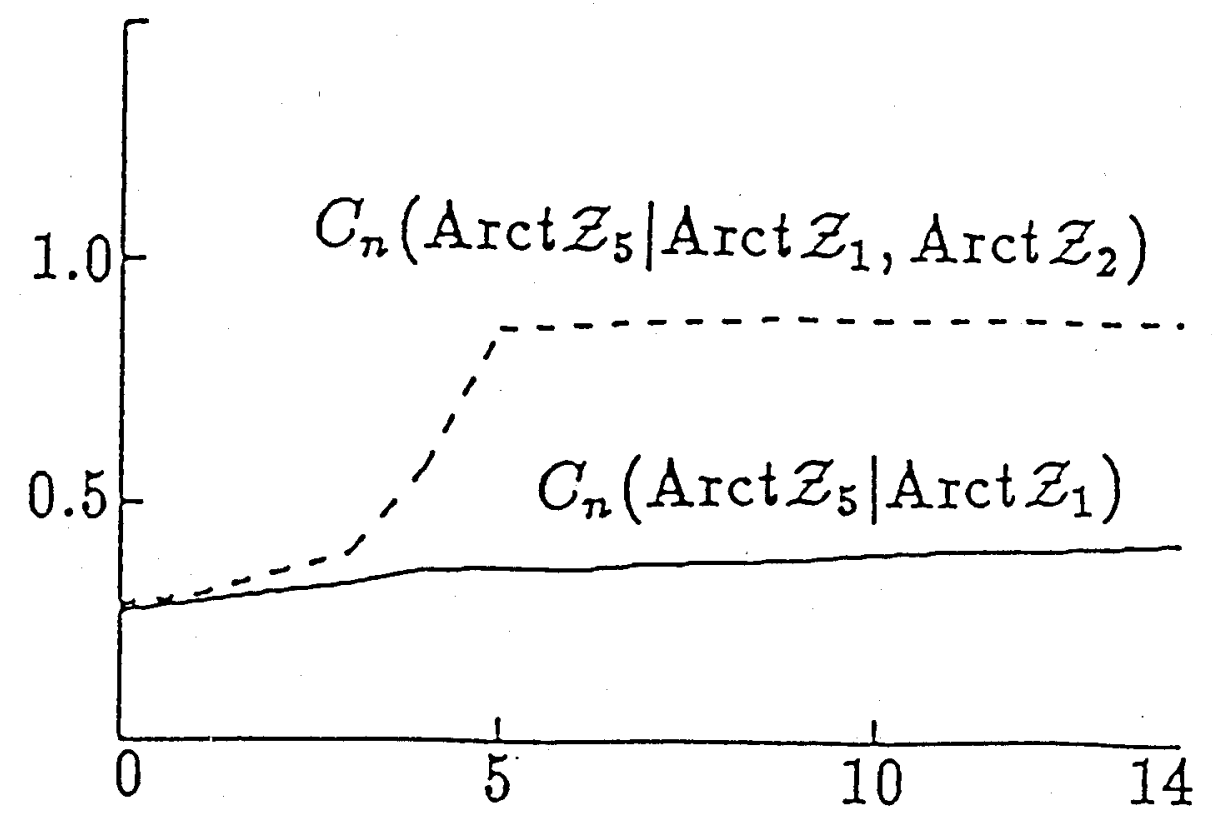

Figure 12.2 Test(CS) 


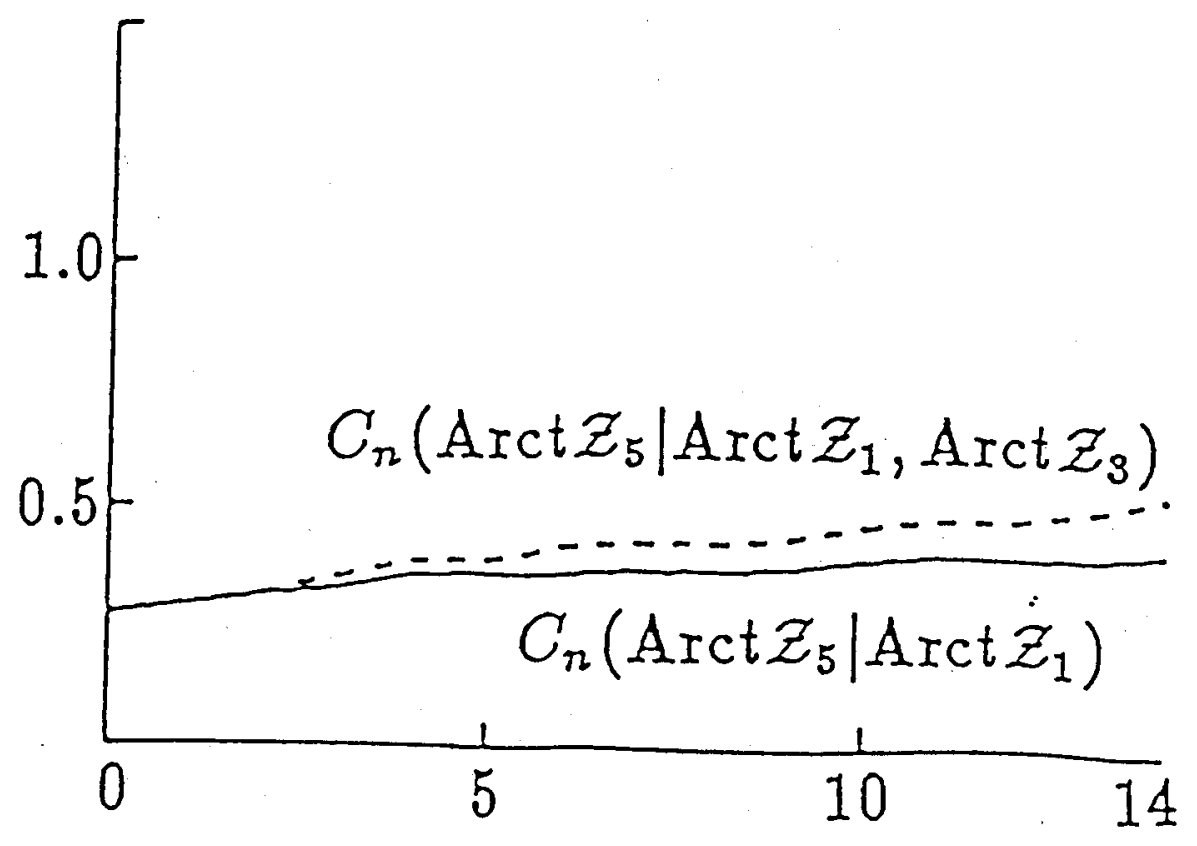

Figure 12.3 Test(CS) 


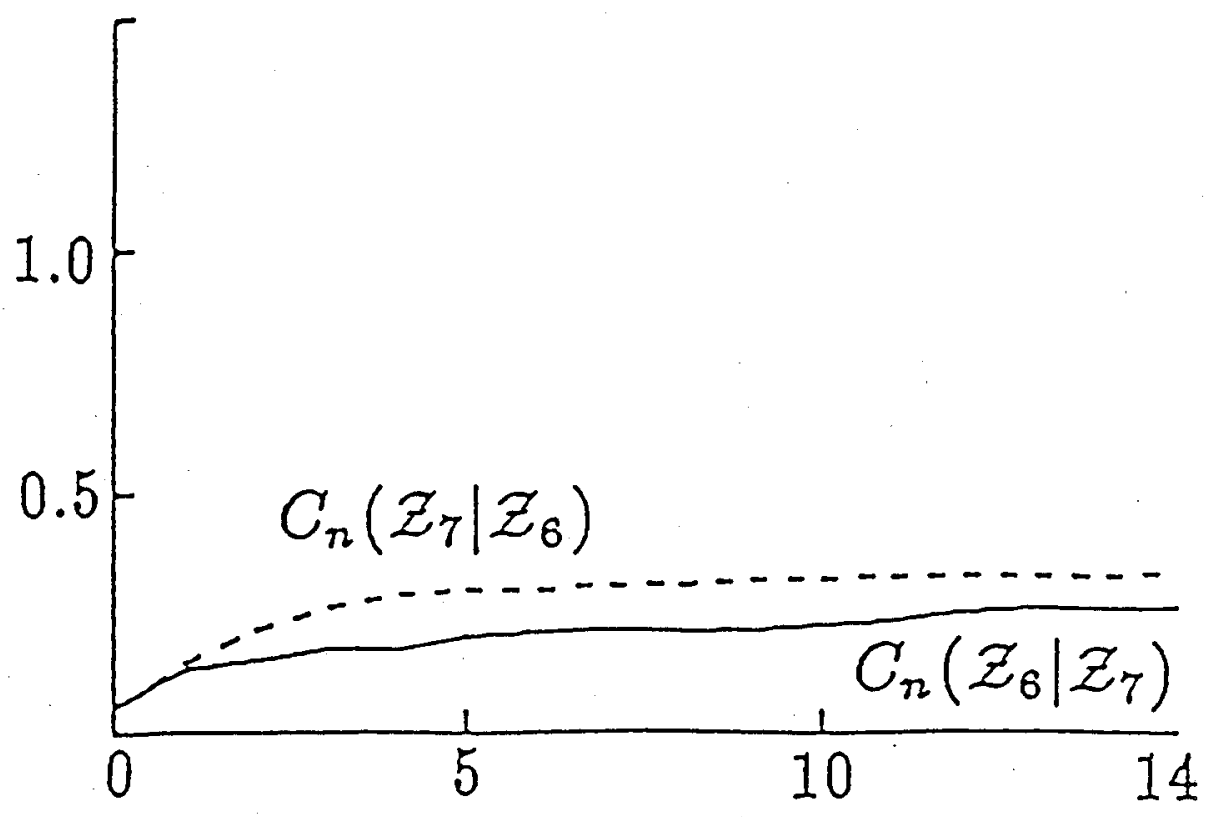

Figure 12.4 Test(CS) 


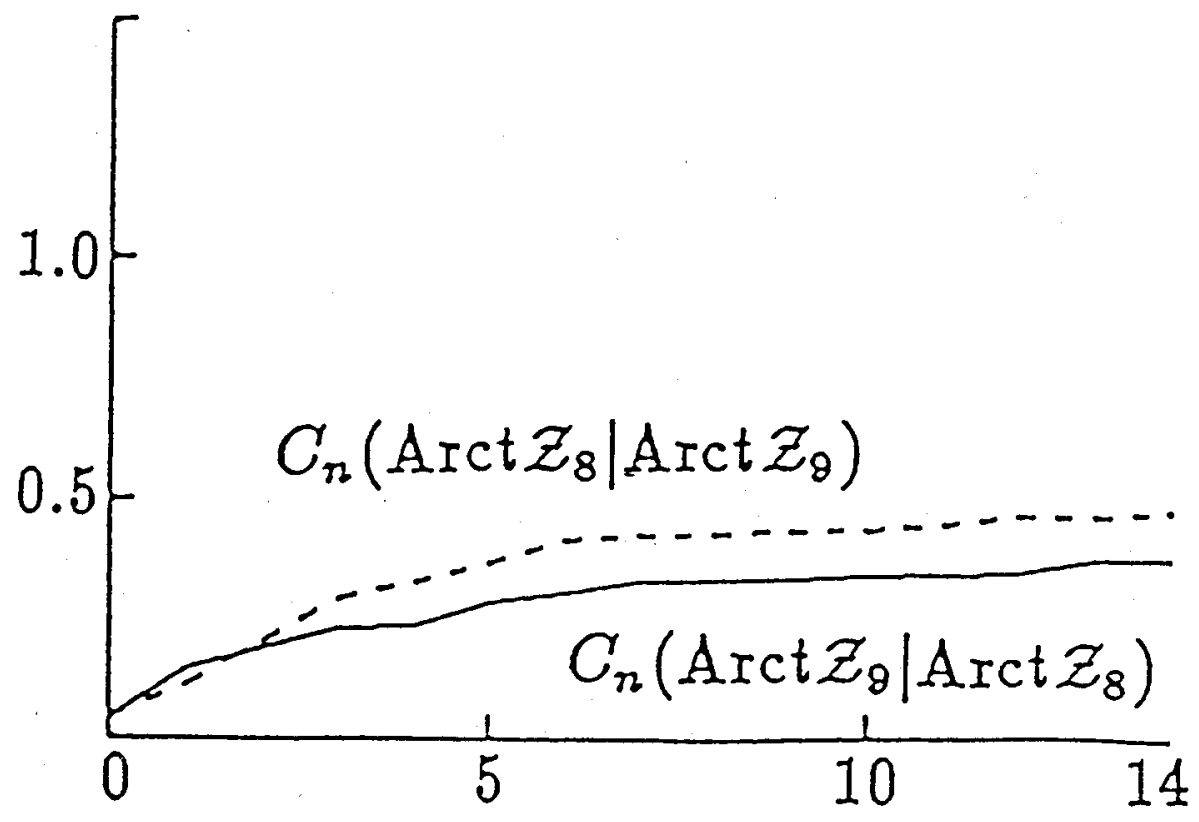

Figure 12.5 Test(CS) 


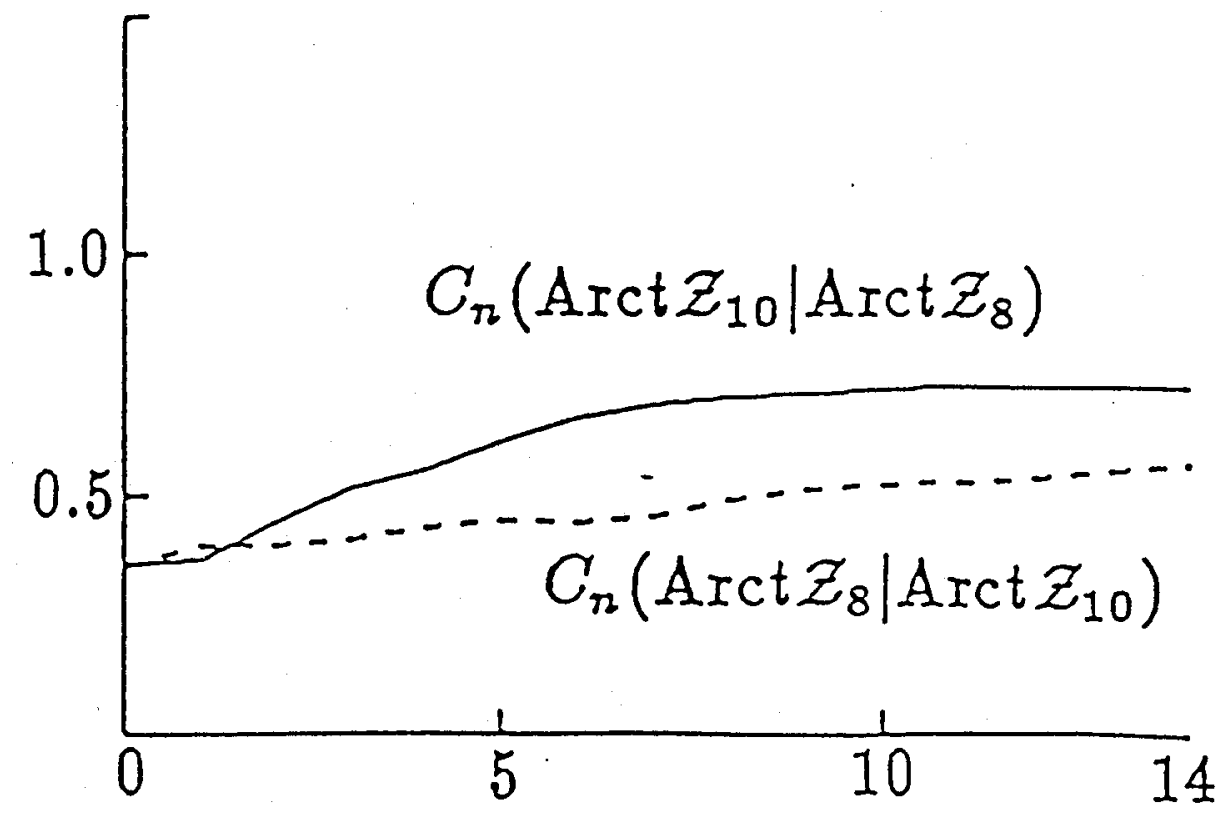

Figure 12.6 Test(CS) 


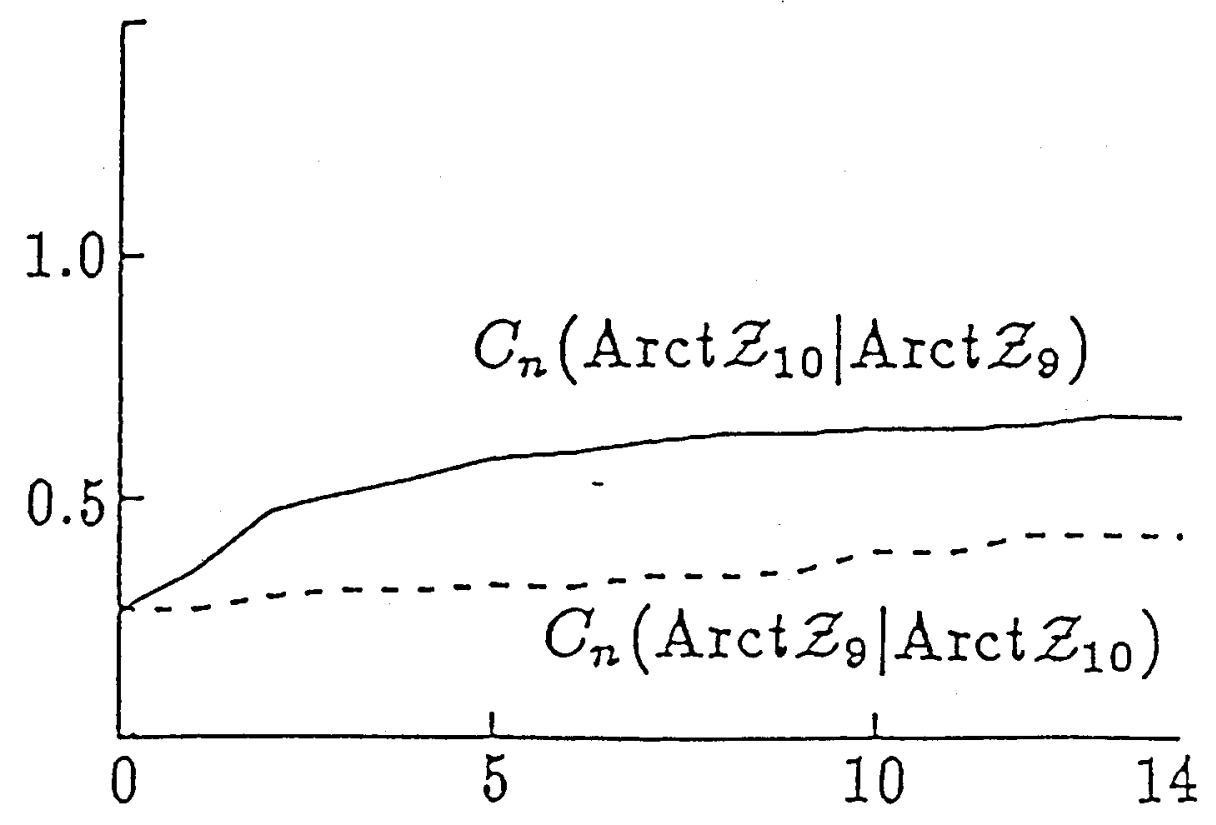

Figure 12.7 Test(CS) 